\title{
VERITAS: the Very Energetic Radiation Imaging Telescope Array System
}

\author{
T.C. Weekes ${ }^{\mathrm{a}, *}$, H. Badran ${ }^{\text {a }}$, S.D. Biller ${ }^{\mathrm{b}}$, I. Bond ${ }^{\mathrm{c}}$, S. Bradbury ${ }^{\mathrm{c}}$, J. Buckley ${ }^{\mathrm{d}}$, \\ D. Carter-Lewis ${ }^{\text {e }}$, M. Catanese ${ }^{\text {a }}, \mathrm{S}$. Criswell ${ }^{\text {a }}$, W. Cui ${ }^{\mathrm{f}}$, \\ P. Dowkontt ${ }^{\text {d }}$, C. Duke ${ }^{\text {g }}$, D.J. Fegan ${ }^{\text {h }}$, J. Finley ${ }^{\text {f }}$, L. Fortson ${ }^{\text {i }}$, J. Gaidos ${ }^{\text {f }}$, \\ G.H. Gillanders ${ }^{\mathrm{j}}$, J. Grindlay ${ }^{\mathrm{a}}$, T.A. Hall ${ }^{\mathrm{e}}$, K. Harris ${ }^{\mathrm{a}}$, A.M. Hillas ${ }^{\mathrm{c}}$, \\ P. Kaaret ${ }^{a}$, M. Kertzman ${ }^{\text {k }}$, D. Kieda ${ }^{1}$, F. Krennrich ${ }^{\text {e }}$, M.J. Lang ${ }^{j}$, \\ S. LeBohec ${ }^{\mathrm{e}}$, R. Lessard ${ }^{\mathrm{f}}$, J. Lloyd-Evans ${ }^{\mathrm{c}}$, J. Knapp ${ }^{\mathrm{c}}$, B. McKernan ${ }^{\mathrm{h}}$, \\ J. McEnery ${ }^{\mathrm{m}}$, P. Moriarty ${ }^{\mathrm{n}}$, D. Muller ${ }^{\mathrm{i}}$, P. Ogden ${ }^{\mathrm{c}}$, R. Ong ${ }^{\mathrm{o}}$, \\ D. Petry $^{\text {e, J. Quinn }}{ }^{\text {h }}$, N.W. Reay ${ }^{\text {p, P.T. Reynolds }}{ }^{\text {q }}$, J. Rose ${ }^{\text {c }}$, M. Salamon ${ }^{1}$, \\ G. Sembroski ${ }^{\text {f }}$, R. Sidwell ${ }^{p}$, P. Slane ${ }^{a}$, N. Stanton ${ }^{p}$, S.P. Swordy ${ }^{i}$, \\ V.V. Vassiliev ${ }^{1}$, S.P. Wakely ${ }^{\mathrm{i}}$ \\ ${ }^{\text {a } W h i p p l e ~ O b s e r v a t o r y, ~ H a r v a r d-S m i t h s o n i a n ~ C e n t e r ~ f o r ~ A s t r o p h y s i c s, ~ P . O . ~ B o x ~ 97, ~ A m a d o, ~ A Z ~ 85645-0097, ~ U S A ~}$ \\ ${ }^{\mathrm{b}}$ Department of Physics, University of Oxford, OX1 3RH, UK \\ ${ }^{\mathrm{c}}$ Department of Physics and Astronomy, University of Leeds, Leeds, LS2 9JT, UK \\ ${ }^{\mathrm{d}}$ Department of Physics, Washington University, Campus Box 1105, One Brookings Drive, St. Louis, MO 63130, USA \\ e Department of Physics and Astronomy, Iowa State University, Ames, IA 50011, USA \\ ${ }^{\mathrm{f}}$ Department of Physics, Purdue University, West Lafayette, IN 47907-1396, USA \\ ${ }^{\mathrm{g}}$ Department of Physics, Grinnell College, Grinnell, IA 50112, USA \\ ${ }^{\mathrm{h}}$ Department of Experimental Physics, National University of Ireland, Dublin, Belfield, Dublin 4, Ireland

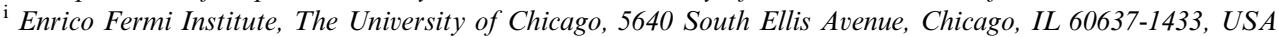 \\ ${ }^{\mathrm{j}}$ Department of Experimental Physics, National University of Ireland, Galway, Ireland \\ ${ }^{\mathrm{k}}$ Department of Physics and Astronomy, DePauw University, Greencastle, IN 46135, USA \\ ${ }^{1}$ Department of Physics, University of Utah, Salt Lake City, UT 84112, USA \\ ${ }^{\mathrm{m}}$ Department of Physics, University of Wisconsin, Madison, WI 53706, USA \\ ${ }^{\mathrm{n}}$ Galway-Mayo Institute of Technology, Galway, Ireland \\ ${ }^{\circ}$ Department of Physics and Astronomy, University of California, Los Angeles, CA 90024, USA \\ ${ }^{\mathrm{p}}$ Department of Physics, Kansas State University, Manhattan, KS 66506-2601, USA \\ ${ }^{\mathrm{q}}$ Cork Institute of Technology, Cork, Ireland
}

Received 14 May 2001; accepted 22 May 2001

\footnotetext{
${ }^{*}$ Corresponding author.

E-mail address: weekes@cfa.harvard.edu (T.C. Weekes).
} 


\begin{abstract}
The Very Energetic Radiation Imaging Telescope Array System (VERITAS) represents an important step forward in the study of extreme astrophysical processes in the universe. It combines the power of the atmospheric Cherenkov imaging technique using a large optical reflector with the power of stereoscopic observatories using arrays of separated telescopes looking at the same shower. The seven identical telescopes in VERITAS, each of aperture $10 \mathrm{~m}$, will be deployed in a filled hexagonal pattern of side $80 \mathrm{~m}$; each telescope will have a camera consisting of 499 pixels with a field of view of $3.5^{\circ}$. VERITAS will substantially increase the catalog of very high energy $(E>100 \mathrm{GeV}) \gamma$-ray sources and greatly improve measurements of established sources. (c) 2002 Elsevier Science B.V. All rights reserved.
\end{abstract}

Keywords: $\gamma$-ray astronomy; $\gamma$-ray telescopes; Atmospheric Cherenkov radiation; Pulsars; AGN; Supernova remnants; Galactic plane; Neutralinos

\section{Introduction}

\subsection{Very high energy $\gamma$-ray astronomy}

Very high energy (VHE) $\gamma$-rays are unique probes of the Universe. Unlike radiation at longer wavelengths, $\gamma$-rays are not attenuated significantly within the Galaxy. As such, they provide an unobstructed view through the plane of the Galaxy except for regions which contain high densities of low energy photons (or virtual photons in regions of high magnetic fields) where VHE $\gamma$-rays are attenuated via photon-photon pair production. The low photon density requirement provides information about possible production sites near active galactic nuclei (AGN) as does the low magnetic field requirement near pulsars. For $\gamma$-rays from extragalactic sources, pair production with low energy photons can be used to probe intergalactic radiation fields. VHE $\gamma$-rays are at the end of the electromagnetic emission spectra of the objects which produce them, so the VHE $\gamma$-ray emission features are often the most sensitive probes of source emission models.

The field of ground-based $\gamma$-ray astronomy has been revolutionized by the development of the atmospheric Cherenkov imaging technique for the discovery and study of individual sources [1-4]. Although less than $1 \%$ of the sky has been surveyed by the imaging technique at energies of 300 $\mathrm{GeV}$ and above, 13 sources have now been reported (eight with high significance) [5]: three pulsar-powered nebulae, six BL Lacertae-type AGN, three shell-type supernova remnants (SNR), and one X-ray binary system. These measurements have advanced our understanding of the origin of cosmic rays, the nature of AGN jets, the density of the background infrared (IR) radiation, and the magnetic fields in the shells and nebulae of SNR.

In this paper the technique and the imperative to progress to a next generation observatory is first described (Section 2). Then the design of the Very Energetic Radiation Imaging Telescope Array System (VERITAS), one such observatory, is presented (Section 3). The technical aspects of VERITAS are described in Section 4. The scientific potential of next generation ground-based telescopes is outlined in Section 5. Finally, in Section 6 the projected sensitivity of VERITAS is compared with other observatories.

\section{The atmospheric Cherenkov imaging technique}

\subsection{Imaging technique}

Atmospheric Cherenkov $\gamma$-ray telescopes have an inherent advantage over space-based $\gamma$-ray telescopes because of their very large collection areas $\left(>40,000 \mathrm{~m}^{2}\right.$ compared with $\left.<1 \mathrm{~m}^{2}\right)$. However, since no anti-coincidence shield can be used in air shower detectors, it is necessary to exploit the differences between $\gamma$-ray showers and hadronic showers to reject the large background of hadronic cosmic rays. Atmospheric Cherenkov imaging was proposed in 1977 for this purpose [6]. The basis of the technique is the use of an array of photomultiplier tubes (PMTs) in the focal plane of 
a large optical reflector to record the Cherenkov image of an air shower. The geometry of the imaging technique is illustrated in Fig. 1.

Since the first experiments, it has been known that there are basic differences in the appearance of the Cherenkov light image of a $\gamma$-ray shower and that of a typical background hadron shower. There is a physical difference in the shower development due to the smaller transverse momentum of electromagnetic interactions compared with hadronic interactions. This difference means that

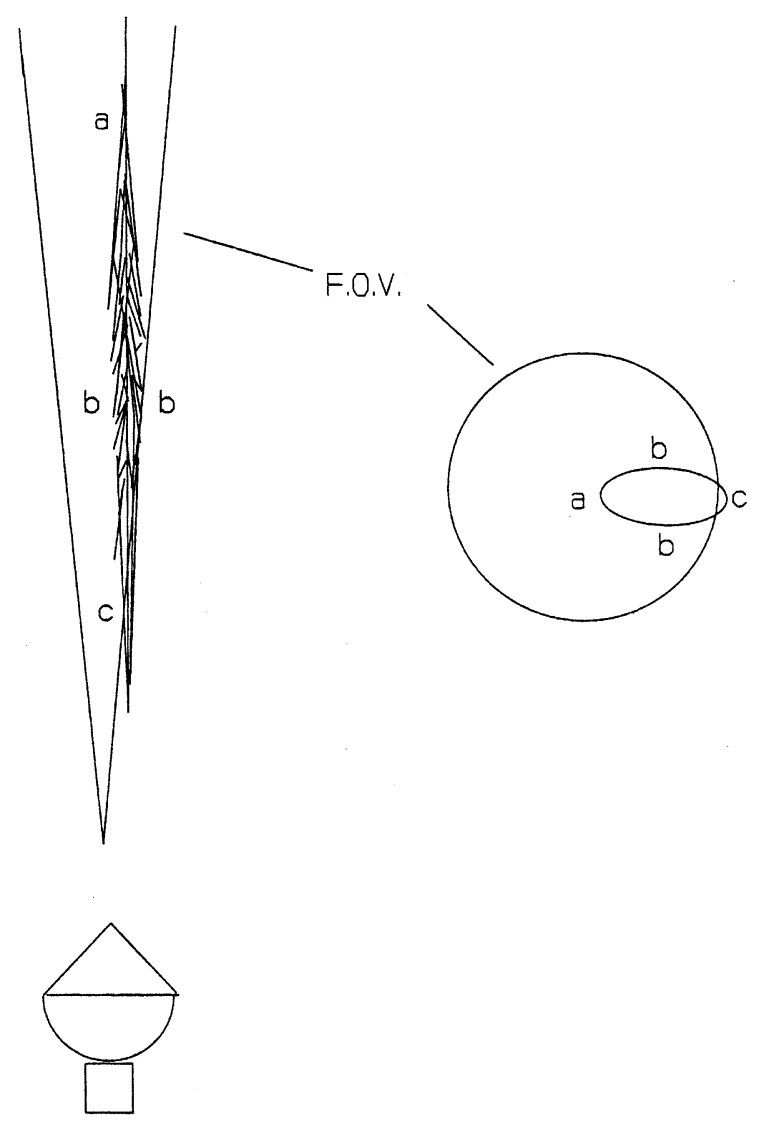

Fig. 1. The imaging technique. The cone of acceptance of the camera intercepts the core of the air shower. The elliptical contour of a typical Cherenkov light image as seen in the focal plane of the camera (typically of diameter $3.5^{\circ}$ ) as seen looking into the camera is shown on the right; note the left to right inversion. Images of $\gamma$-ray showers coming from a source parallel to the optic axis are narrow and point towards the center. Images from background cosmic rays are broader and have no preferred pointing direction. the Cherenkov radiating particles in the $\gamma$-ray shower are, on average, closer to the direction of the primary. In addition there is no penetrating component in electromagnetic showers, so the local contribution from individual particles to the light is small and the fluctuations in the shower image are less. Because the $\gamma$-ray images are better defined, it is easier to characterize their arrival direction and hence to discriminate them against the isotropic background of hadron showers.

The power of the imaging technique was first demonstrated using an array of PMTs in the focal plane of the Whipple Observatory $10 \mathrm{~m}$ optical reflector [7]; the detection of the Crab Nebula was the first major success of the technique $[8,9]$. The background was rejected with $99.7 \%$ efficiency and the source location was determined with an accuracy of $0.05^{\circ}$. The Whipple camera design has since been duplicated at a number of observatories [10-17] where it has been successfully used to extend the catalog of detected $\mathrm{TeV}$ sources.

\subsection{Imaging with arrays}

The atmospheric Cherenkov imaging technique can be significantly improved by the use of multiple telescopes with separations of the same order as the lateral spread of the light from the shower. The technique has been effectively demonstrated using pairs of telescopes [18] and arrays [19]. The reduction in energy threshold is less than that achieved by combining all the mirror area into one telescope; however the distribution of mirror area into multiple telescopes gives many images of the same shower which offer the following advantages:

- improved hadron discrimination from multiple image characterization,

- elimination of local muon background,

- improved energy resolution from multiple measurements, shower axis location and determination of shower maximum,

- improved angular resolution.

\subsection{Next generation telescopes}

Advances made by the present generation of imaging telescopes justify the construction of an 
array of large imaging telescopes with the following properties:

- large effective area: $>0.1 \mathrm{~km}^{2}$ to provide sensitive measurements of short variability timescales,

- better flux sensitivity: detection of sources which emit $\gamma$-rays at levels of $0.5 \%$ of the Crab Nebula flux at energies of $300 \mathrm{GeV}$ in $50 \mathrm{~h}$ of observation,

- reduced energy threshold: an effective energy threshold $<100 \mathrm{GeV}$ with significant sensitivity at $50 \mathrm{GeV}$,

- improved energy resolution: a root mean square (RMS) spectral resolution of $\Delta E / E<0.10-0.15$ for an individual shower over a broad energy range $(E>300 \mathrm{GeV})$,

- increased angular resolution: $<0.05^{\circ}$ for individual showers and source location better than $0.005^{\circ}$ (>100 photons),

- large field of view: at least $3^{\circ}$ diameter as used in many current atmospheric Cherenkov imaging telescopes.

All of these objectives can be achieved by VERITAS, an array of large imaging telescopes which are improved versions of the existing Whipple 10 $\mathrm{m}$ imaging telescope [20]. The seven telescopes in VERITAS will be identical and will be deployed as shown in Fig. 2. Six telescopes will be located at the corners of a hexagon of side $80 \mathrm{~m}$ and one will be located at the center. The telescopes will each have a camera consisting of 499 pixels with a field of view of $3.5^{\circ}$ diameter.

The VERITAS concept was first described in 1985 prior to the definite detection of any $\mathrm{TeV}$ sources [21]. The first practical demonstration of the power of an array of imaging telescopes came with the completion of HEGRA, an array of five $3.5 \mathrm{~m}$ aperture telescopes [19].

\section{VERITAS design}

The performance characteristics required of VERITAS are derived from its scientific goals which are discussed in Section 5. VERITAS was designed to optimize observations of a variety of

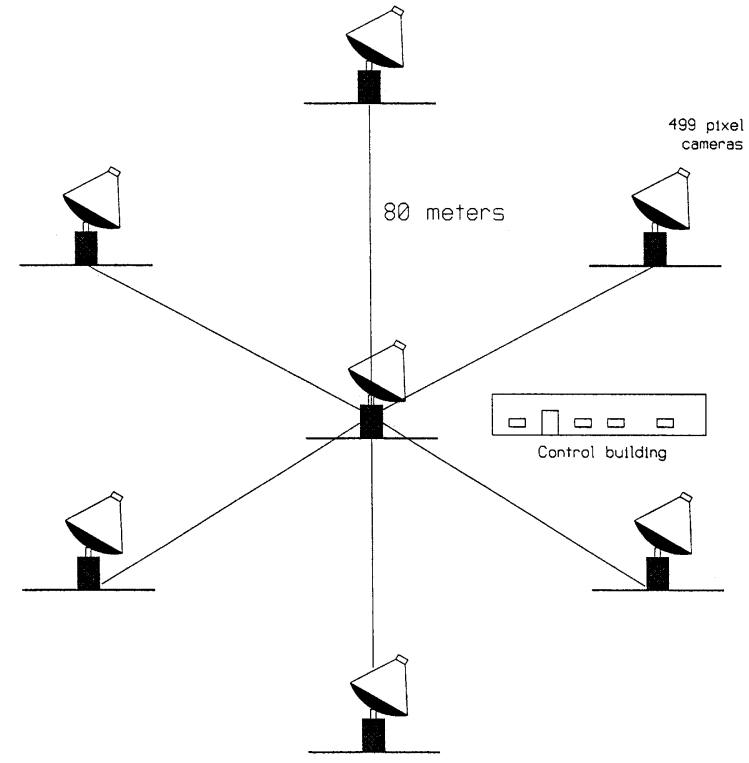

Fig. 2. Layout of telescopes in VERITAS.

sources, each of which may require a different subset of capabilities (e.g., continuous monitoring, large field of view (FOV), good flux sensitivity, large collection area, accurate angular resolution, low energy threshold, accurate energy resolution, prompt response, and broad energy coverage). With these features, VERITAS can observe known and anticipated sources in several observing modes.

Extensive simulation studies have been used to determine the optimum configuration for VERITAS and to characterize its performance; these are described in detail elsewhere [22-24]. The VERITAS design is optimized for maximum sensitivity to point sources in the energy range $100 \mathrm{GeV}-10$ $\mathrm{TeV}$, but with significant sensitivity in the range 50-100 GeV and from 10-50 TeV.

\subsection{Choice of VERITAS parameters}

To determine the optimum array configuration, several parameters were varied: the number of telescopes, the spacing between the telescopes, the focal length of the telescope, the aperture of the telescopes, and the FOV of the cameras. The number of channels in the camera was fixed before 
the optimization for cost reasons. Thus, the investigations of different camera fields of view are simultaneously investigations of changes in the spacing of the PMTs. In the medium energy range of $200 \mathrm{GeV}-1 \mathrm{TeV}$, the sensitivity of the array to point sources is not significantly affected by small changes in the array's characteristics. The choice of telescope parameters was driven mostly by their effects on the performance of VERITAS at the low and high ends of the sensitive energy range.

\subsubsection{Number of telescopes}

At a minimum, three telescopes are necessary to utilize the stereoscopic imaging technique. Two telescopes can be used for bright images, but faint cascades can only be accurately reconstructed with images in three telescopes because the individual image axes are not well defined. There are three primary reasons for choosing a seven telescope array over one with three to four telescopes with similar characteristics.

1. Low energies: Although high sensitivity measurements in the energy region above $130 \mathrm{GeV}$ will be accessible with a smaller array of $10 \mathrm{~m}$ telescopes (e.g., three or four telescopes), the energy range from 50 to $130 \mathrm{GeV}$ is only accessible with the full array of seven telescopes. Some cosmic sources (e.g., $\gamma$-ray pulsars or possibly $\gamma$-ray bursts (GRB)) may be detectable only in the lowest energy range. However, energy flux measurements on all sources will benefit from having well-defined spectral measurements over a wide range of energies. The differential flux sensitivity is defined as the flux level in an energy interval of $1 / 4$ of a decade over which an energy flux measurement can be made with less than $20 \%$ error in $5 \mathrm{~h}$ of observation. This is the meaningful level for astrophysical measurements. The flux sensitivity for the full array is shown in Fig. 3 where it is contrasted with the flux sensitivity for a three telescope array (sub-array). For reference, the measured spectrum of Markarian 421 [25] observed during a high state of emission is also shown.

2. Versatility: The observing program of VERITAS [24] can only be accomplished in a timely fashion if the array can be divided in two or more sub-arrays for simultaneous monitoring of different regions of

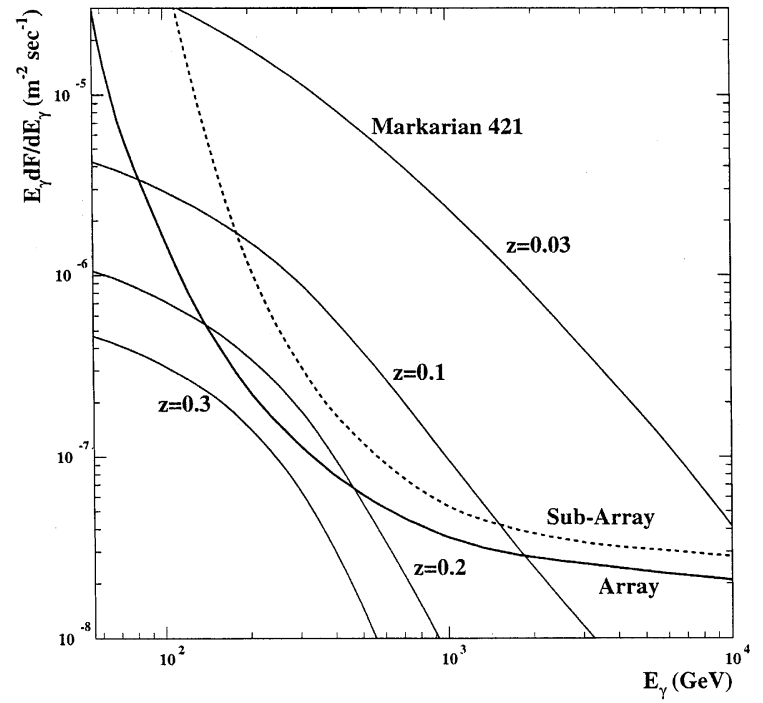

Fig. 3. The differential flux sensitivity of full array and subarray (three telescopes) of VERITAS is shown as a function of energy. The measured spectrum of Mrk 421 in flaring state is shown $(z=0.03)$; the spectra of the same source if it were at $z=0.1,0.2$ and 0.3 are also shown.

the sky. A sub-array of three or four telescopes is very powerful and performs almost as well as the full array for the detection of new sources and measurement of established sources above $\sim 250$ $\mathrm{GeV}$ (although only the full array will reach the lowest energies). Above $\sim 250 \mathrm{GeV}$, a sub-array also has angular resolution comparable to the full array and provides excellent performance for spectral studies. Some scientific studies, such as the long-term variability of AGN, can only be accomplished with a sub-array dedicated to monitoring one particular object for extensive time periods. It is anticipated that the array will be operated as two independent sub-arrays at least $50 \%$ of the time.

3. Flexibility: Experience has shown that the full potential of an atmospheric Cherenkov telescope is only realized after the detection of $\gamma$-ray sources. Therefore, it is anticipated that, with operating experience, significant improvements will be made in the achievable flux sensitivity of VERITAS by varying the observing techniques, the telescope hardware, and the data analysis algorithms. The multi-telescope configuration of VERITAS presents the maximum adaptability in this regard. 


\subsubsection{Separation of telescopes}

The optimal separation between the telescopes was chosen to be $80 \mathrm{~m}$. This provides the best combination of flux sensitivity and energy threshold. Increasing the spacing does not improve flux sensitivity in the $200 \mathrm{GeV}-1 \mathrm{TeV}$ energy range but it does increase the energy threshold. Decreasing the spacing can reduce the energy threshold somewhat, but the sensitivity is significantly reduced over the entire energy range because the stereoscopic capabilities become less efficient and the angular resolution degrades leading to a dramatic increase in the number of unrejected background events.

\subsubsection{Optical design}

The chosen FOV of the telescope is $3.5^{\circ}$. From experience operating the Whipple telescope, this is the minimum required for individual telescopes to reconstruct accurately images from point sources and it also gives significant sensitivity to off-axis or extended sources. Increasing the FOV beyond this will improve the high energy response of the telescope, but also increases the energy threshold for the fixed number of channels. The chosen FOV is a compromise between these competing priorities.

The focal length chosen for the telescopes is 12 $\mathrm{m}$, which makes them $f / 1.2$ systems for an aperture of $10 \mathrm{~m}$. Increasing the focal length from the $f / 0.7$ of the Whipple $10 \mathrm{~m}$ significantly improves the optical quality of the telescopes by reducing the effects of aberrations. This improvement is necessary to match the angular size of the PMTs in the VERITAS cameras $\left(\sim 0.15^{\circ}\right)$ while retaining the $3.5^{\circ} \mathrm{FOV}$. However, increasing the focal length beyond $12 \mathrm{~m}$ does not improve the performance of VERITAS enough to justify the increased cost and complexity needed to achieve the required alignment precision and stability for the pixel size chosen. Also, the reduction in energy threshold for increasing the focal length beyond $12 \mathrm{~m}$ is outweighed by the less efficient light collection caused by increasing the pixel separation to maintain the required FOV. Thus, $12 \mathrm{~m}$ provides the best combination of improved optical quality, sufficient FOV and small pixel spacing.

\subsubsection{Trigger}

The telescope trigger requires three adjacent PMTs to record more than 4.2 photoelectrons (p.e.) within $\approx 10$ ns. The 4.2 p.e. trigger threshold level is set by the sustainable single telescope trigger rate at which VERITAS can operate $(1 \mathrm{MHz})$ and by the requirement that the array trigger rate from fluctuations of the night-sky background (NSB) not exceed $10 \%$ of the total cosmic-ray rate of $1 \mathrm{kHz}$.

\subsection{Collection area}

The collection area of the array reflects a combination of the area over which the array can detect $\gamma$-ray showers and the efficiency for retaining those events after analysis. The effective collection area of VERITAS as a function of photon energy is shown in Fig. 4 for the PMT trigger threshold of 4.2 p.e. For comparison, the effective area of the Whipple telescope near $1 \mathrm{TeV}$ is $\approx 4 \times 10^{4} \mathrm{~m}^{2}$ [27]. The effective collection area at low energies is reduced because these showers emit fewer Cherenkov photons and so must arrive with optimum geometry to trigger the telescope and allow for event reconstruction. For the highest energies, the

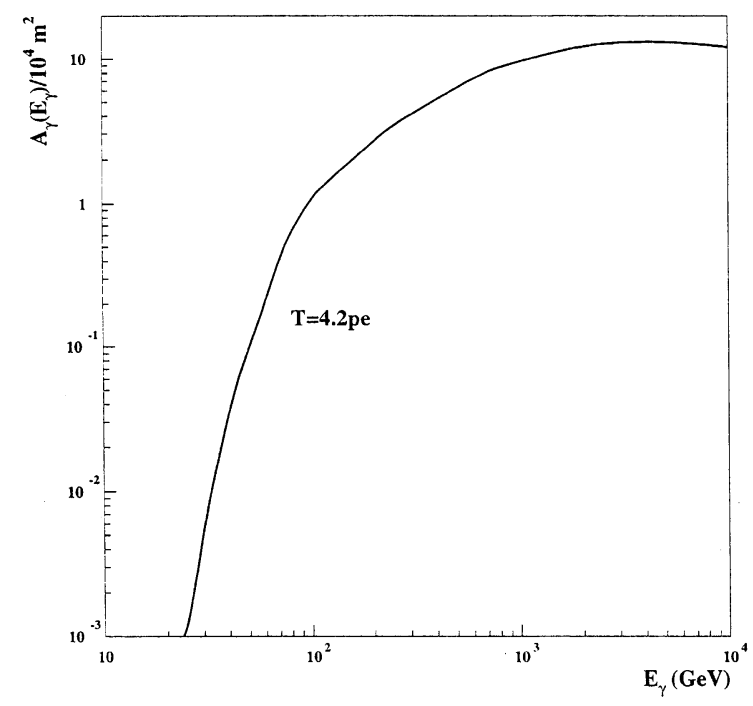

Fig. 4. The collection area of VERITAS as a function of $\gamma$-ray energy. 
collection area levels out because the limited FOV of the telescope truncates these images which develop low in the atmosphere.

\subsection{Energy threshold}

The peak energy of VERITAS is defined as the energy at which the differential rate of reconstructed $\gamma$-rays from the Crab Nebula per unit interval of energy reaches its maximum. Reconstructed $\gamma$-rays are those whose images pass an image cleaning procedure (to eliminate pixels whose light is dominated by fluctuations of the NSB light), a reconstructed direction cut, and an image shape cut. This rate curve is shown in Fig. 5 for a 4.2 p.e. three adjacent pixel trigger threshold and indicates a array peak energy of $\approx 75 \mathrm{GeV}$. This array peak energy is substantially higher than the trigger peak energy i.e., the peak in the $\gamma$-ray rate for events triggering the telescope, because low energy Cherenkov events which trigger the telescope often do not produce enough light to be used in the analysis. However, events at energies below the array peak energy which trigger

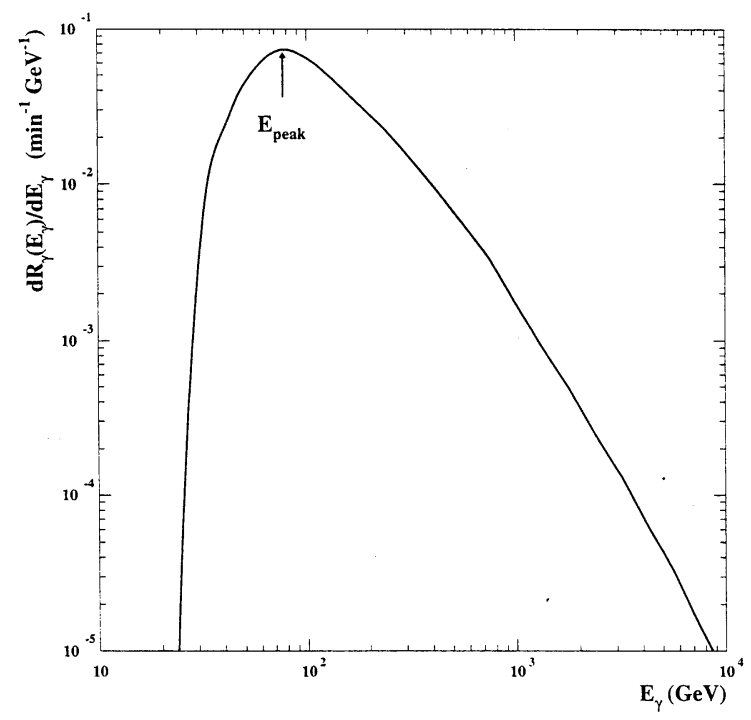

Fig. 5. Differential detection rates of the Crab Nebula for VERITAS when the telescope trigger is from three adjacent pixels. The peak of the curve is sometimes referred to as the energy threshold (see text) but here is called the peak energy. the array can still be successfully reconstructed, as shown by the extent of the rate curves below the peak. Strong sources with spectral cut-offs below the array peak energy may still be detectable.

A peak energy of $75 \mathrm{GeV}$ is a factor of 4 lower than that reached by existing imaging Cherenkov telescopes. This improvement comes from the combination of the increase in mirror area, the stereoscopic reconstruction of events, which permits effective background rejection at lower light levels than a single telescope, and the multi-telescope trigger, which permits the operation of the individual telescopes much further into the region where fluctuations in the NSB dominates the single telescope trigger rate.

The conventional definition of the energy threshold is the point where the differential rate of $\gamma$-rays per unit energy interval from the Crab Nebula is the highest; we prefer to call this the peak energy since the actual threshold may be considerably below this level (Fig. 5).

\subsection{Energy resolution}

The energy resolution of VERITAS will be considerably better than that of a single atmospheric imaging telescope such as the Whipple telescope because (1) the shower core location will be known with an accuracy of a few meters, and (2) several telescopes will view each event at different distances from the shower core. With a single telescope, the core distance can be measured using the location of the image centroid relative to the source position, but the correlation is not very tight and the corresponding energy estimate is not very accurate. For the Whipple telescope, the RMS energy resolution using this technique is $\Delta E / E \approx 0.35$ [28].

The overall energy RMS resolution of VERITAS will be $\Delta E / E \approx 0.10-0.15$ from 0.2 to $10 \mathrm{TeV}$. The resolution improves slowly as the energy of the shower increases. Below $200 \mathrm{GeV}$, the energy resolution degrades. This low energy resolution will likely be improved through more sophisticated energy estimates and through the use of more restrictive cuts on events used in the spectral analysis. 


\subsection{Angular resolution}

The angular resolution is defined as the width of a two-dimensional Gaussian fit to the distribution of reconstructed directions for individual photons from a point source [24]. The angular resolution of VERITAS for a single photon as a function of peak energy is shown in Fig. 6. It is convenient to characterize integral parameters of the array, such as angular resolution or flux sensitivity, as functions of array peak energy. The majority of events reconstructed with a given angular resolution will correspond to $\gamma$-rays with energies equal to the peak energy. VERITAS will have better angular resolution than any existing detector (in space or on the ground) operating above a few $\mathrm{MeV}$. The main reason for the excellent angular resolution of VERITAS is its stereoscopic imaging capability. For a single telescope, the photon arrival direction is well defined only in the direction perpendicular to the axis of the image, though the direction parallel to the direction of the image axis can be roughly estimated [26]. Multiple sampling of the shower from the VERITAS telescopes overcomes this difficulty and, for larger showers, can average out fluctuations. The improvement in angular resolution alone leads to better background rejec-

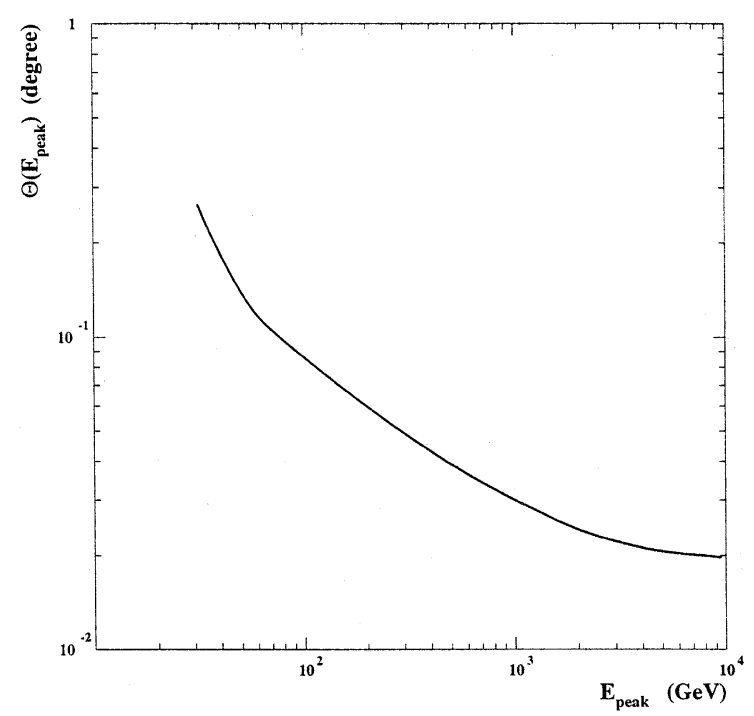

Fig. 6. Angular resolution of VERITAS for a single photon as a function of array $\gamma$-ray peak energy. tion than existing single telescopes and it also will allow the emission regions of extended sources to be mapped to arc-minute accuracy. In the absence of test beam, the angular resolution will be verified by observations of a point source, e.g., AGN.

\subsection{Integral flux sensitivity}

The performance of VERITAS is summarized by its $\gamma$-ray flux sensitivity versus energy. The minimum detectable flux of $\gamma$-rays is defined by the confidence level required for detection or by the statistics of the detected photons. A $5 \sigma$ excess of $\gamma$-rays above the background, or 10 photons (below this, Poisson statistics must be used) is required. The flux sensitivity is estimated for $50 \mathrm{~h}$ of observations on an object with a differential spectrum given by $\mathrm{d} N / \mathrm{d} E \propto E^{-2.5}$, as seen from the Crab Nebula in this energy range. Direction and image shape cuts are applied to reject background events and optimize sensitivity.

This conservative estimate of the $\gamma$-ray integral flux sensitivity of VERITAS for point sources as a function of array energy threshold is shown in Fig. 7. The complex shape of the sensitivity curve is caused by different energy regions being dominated by the different backgrounds shown. For energies above 2-3 TeV, the sensitivity of VERITAS is limited by photon statistics. Larger telescope FOVs can improve this sensitivity in the future, as can large zenith angle observations. In the region near $1 \mathrm{TeV}$, the sensitivity is limited by those rare cosmic-ray protons which mimic $\gamma$-rays by converting most of their energy into an electromagnetic cascade in the first few interactions (prompt decay of $\pi^{0}$ ). In the energy region between $200 \mathrm{GeV}$ and $\sim 1 \mathrm{TeV}$, the background rejection of VERITAS is so good that diffuse cosmic-ray electrons are expected to dominate the background instead of hadronic cosmic rays. The region below $200 \mathrm{GeV}$ is limited by hadronic cosmic-ray events whose reconstruction is significantly affected by small NSB fluctuations. The lower curve indicates a relatively dark observation region (near the zenith with no bright stars in the FOV) while the upper curve indicates a region where the NSB light is approximately four times brighter (as in some regions of the Galactic plane). 


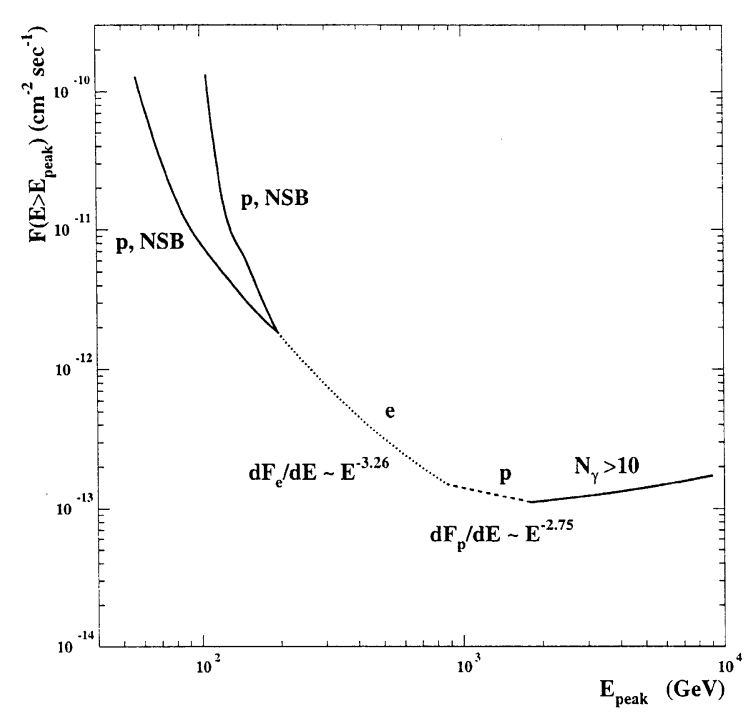

Fig. 7. The $\gamma$-ray sensitivity of VERITAS to point-like sources in $50 \mathrm{~h}$ of observing. The dominant background as a function of energy threshold is indicated (see text for details). The two curves at low energies indicate the sensitivity of VERITAS in dark (lower curve) and bright (upper curve) NSB regions. P refers to the hadronic background, e to the electron background. A minimum of ten photons is required at high energies. These are the sensitivities at small zenith angles; at large zenith angles the sensitivity improves for higher energies.

In Table 1 the performance characteristics of VERITAS are summarized; these are optimized for point source detection.

\section{Technical description}

\subsection{Telescope design}

The Davies-Cotton optical design [29] used in the Whipple $10 \mathrm{~m}$ reflector (and in many subsequent $\gamma$-ray telescopes) has mirror facets which are spherical and identical, facilitating fabrication at a reasonable cost and making alignment easy. This design also has smaller off-axis aberrations than a parabolic reflector so that it has good image quality out to a few degrees from the optic axis. Its one limitation, that the surface is not isochronous, means that the reflector introduces a time spread into the light pulse. Alternative designs were investigated but it was concluded that the DaviesCotton collector provides the optimal combination
Table 1

VERITAS sensitivity

\begin{tabular}{|c|c|c|}
\hline Characteristic & $E$ & Value \\
\hline Peak energy ${ }^{a}$ & & $75 \mathrm{GeV}$ \\
\hline Flux sensitivity ${ }^{b}$ & $\begin{array}{l}>100 \mathrm{GeV} \\
>300 \mathrm{GeV} \\
>1 \mathrm{TeV}\end{array}$ & $\begin{array}{l}9.1 \times 10^{-12} \mathrm{~cm}^{-2} \mathrm{~s}^{-1} \\
=15 \mathrm{mCrab} \\
8.0 \times 10^{-13} \mathrm{~cm}^{-2} \mathrm{~s}^{-1} \\
=5 \mathrm{mCrab} \\
1.3 \times 10^{-13} \mathrm{~cm}^{-2} \mathrm{~s}^{-1} \\
=7 \mathrm{mCrab}\end{array}$ \\
\hline Angular resolution & $\begin{array}{l}50 \mathrm{GeV} \\
100 \mathrm{GeV} \\
300 \mathrm{GeV} \\
1 \mathrm{TeV}\end{array}$ & $\begin{array}{l}0.14^{\circ} \\
0.09^{\circ} \\
0.05^{\circ} \\
0.03^{\circ}\end{array}$ \\
\hline Effective area & $\begin{array}{l}50 \mathrm{GeV} \\
100 \mathrm{GeV} \\
300 \mathrm{GeV} \\
1 \mathrm{TeV}\end{array}$ & $\begin{array}{l}1.0 \times 10^{3} \mathrm{~m}^{2} \\
1.0 \times 10^{4} \mathrm{~m}^{2} \\
4.0 \times 10^{4} \mathrm{~m}^{2} \\
1.0 \times 10^{5} \mathrm{~m}^{2}\end{array}$ \\
\hline $\begin{array}{l}\text { Crab Nebula } \\
\gamma \text {-ray rates } \\
\text { Energy resolution }^{\mathrm{c}}\end{array}$ & $\begin{array}{l}>100 \mathrm{GeV} \\
>300 \mathrm{GeV} \\
>1 \mathrm{TeV}\end{array}$ & $\begin{array}{l}50 / \mathrm{min} \\
7 / \mathrm{min} \\
1 / \mathrm{min} \\
<15 \%\end{array}$ \\
\hline
\end{tabular}

${ }^{\text {a }}$ Energy at which the rate of photons per unit energy interval from the Crab Nebula is highest for a 4.2 p.e. trigger threshold.

${ }^{\mathrm{b}}$ Minimum integral flux for a $5 \sigma$ excess (or $\geqslant 10$ events) in 50 $\mathrm{h}$ of observations of a source with a Crab Nebula-like spectrum.

${ }^{\mathrm{c}} \mathrm{RMS}(\Delta E / E)$.

of optical quality and cost effectiveness. Increasing the $f$-number from $f / 0.7$ to $f / 1.2$ substantially improves the optical quality of the telescope (Fig. $8)$. It is then possible to match the inherent fluctuations in the shower images with a camera that has a reasonable number of pixels $(\approx 500)$ and appropriate FOV $\left(\approx 3.5^{\circ}\right)$. The larger $f$ /number reduces the time spread to $<4$ ns (which is a good match to the inherent width of the shower pulse).

The desirable optical parameters of the telescope follow from the characteristics of a $\gamma$-ray shower Cherenkov image. For a $\gamma$-ray source at the center of the FOV, the average image centroid (distance) is $\approx 0.85^{\circ}$ from the center of the focal plane, and the centroid moves to slightly larger values at larger energies. The image has a RMS width and length of about $0.14^{\circ}$ and $0.25^{\circ}$, respectively. The reflector will have sufficient resolution to record image structure on this scale. 

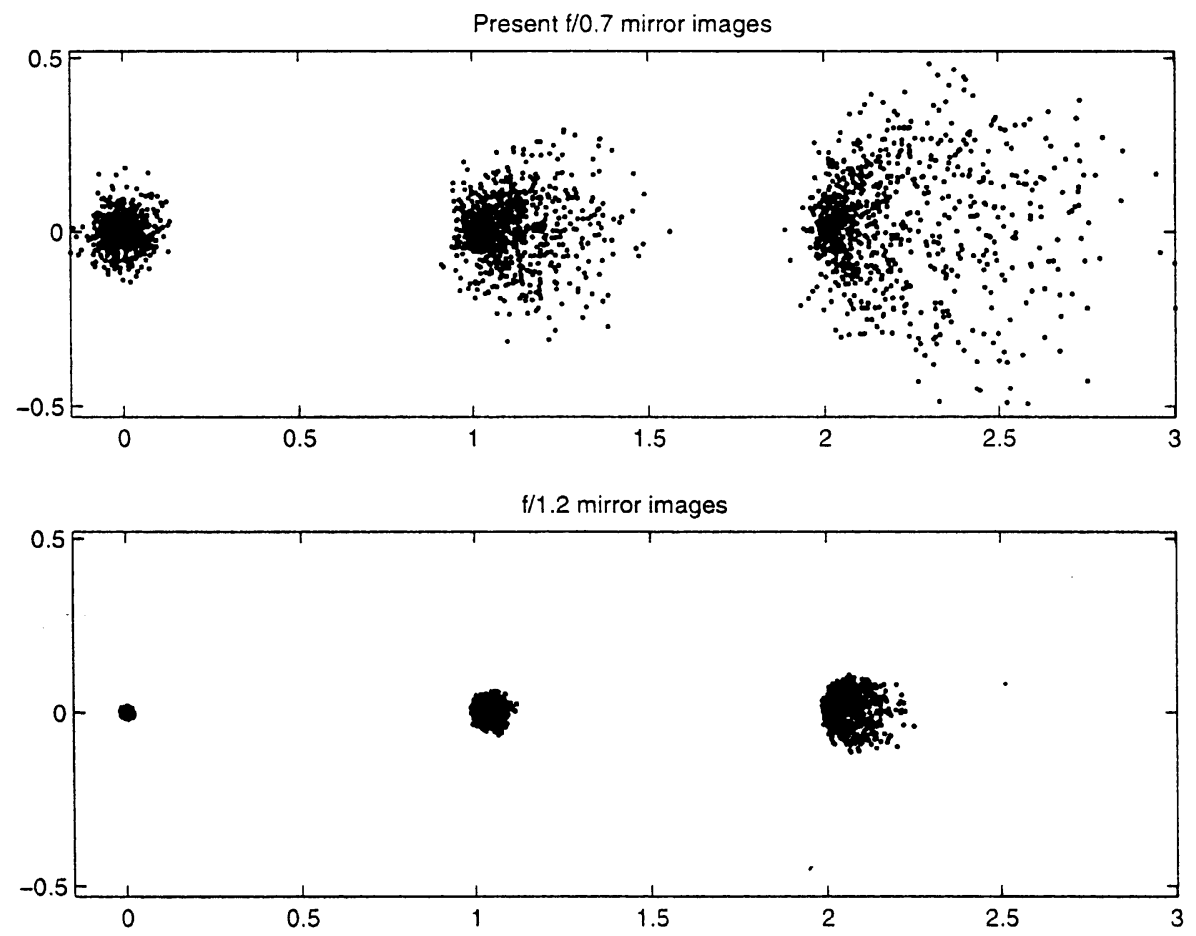

Fig. 8. Point spread images in the focal plane corresponding to a source at $0^{\circ}, 1^{\circ}$, and $2^{\circ}$ off axis for $f /$ numbers of 0.7 (top, Whipple telescope) and 1.2 (bottom, VERITAS). The facet size is $60 \mathrm{~cm}$ and the image size is given in degrees. A total of 1000 points lie in each image.

A key factor in the reflector quality and cost is the mirror facets. Although a number of mirror materials and fabrication technologies have been developed and investigated for VERITAS, a conventional approach has been chosen as the most cost effective. The facets will be made of float glass, ground or slumped, polished, aluminized and anodized. They will be hexagonal in shape to permit closepacking and will be $60 \mathrm{~cm}$ across the flats.

\subsection{Mounts: Optical support structurelpositioner}

The VERITAS telescopes will duplicate the Whipple telescope in using a custom-designed, welded-steel, space-frame optical support structure (OSS) mounted on a commercial positioner. They will have a $f /$ number of 1.2 , a rectangular OSS to facilitate the use of mirror covers, and tighter performance specifications than the Whipple $10 \mathrm{~m}$ reflector.
Simpson, Gumpertz and Heger (SG\&H), the firm that designed the Whipple $10 \mathrm{~m}$ telescope, have conducted two studies and a conceptual design. Cost and performance were evaluated as the critical parameters of aperture, $f$-number, wind loading, storm survival, stiffness, and pointing were varied. Based on these studies, SG\&H determined that a trussed-steel OSS of $10 \times 11 \mathrm{~m}$ aperture and $12 \mathrm{~m}$ focal length $(f / 1.2)$ could be designed to match commercially available positioners (Fig. 9). Although initially the OSS will have 250 mirrors (collection area $=75 \mathrm{~m}^{2}$ ), it will be capable of supporting 315 mirrors (collection area $=100 \mathrm{~m}^{2}$ ). SG\&H confirmed that a $f / 1.2$ OSS can be constructed locally from standard materials to meet VERITAS design specifications.

The telescope RMS blur, RMS decentering, and RMS pointing specifications, with and without corrections (determined by offset-facet alignment 


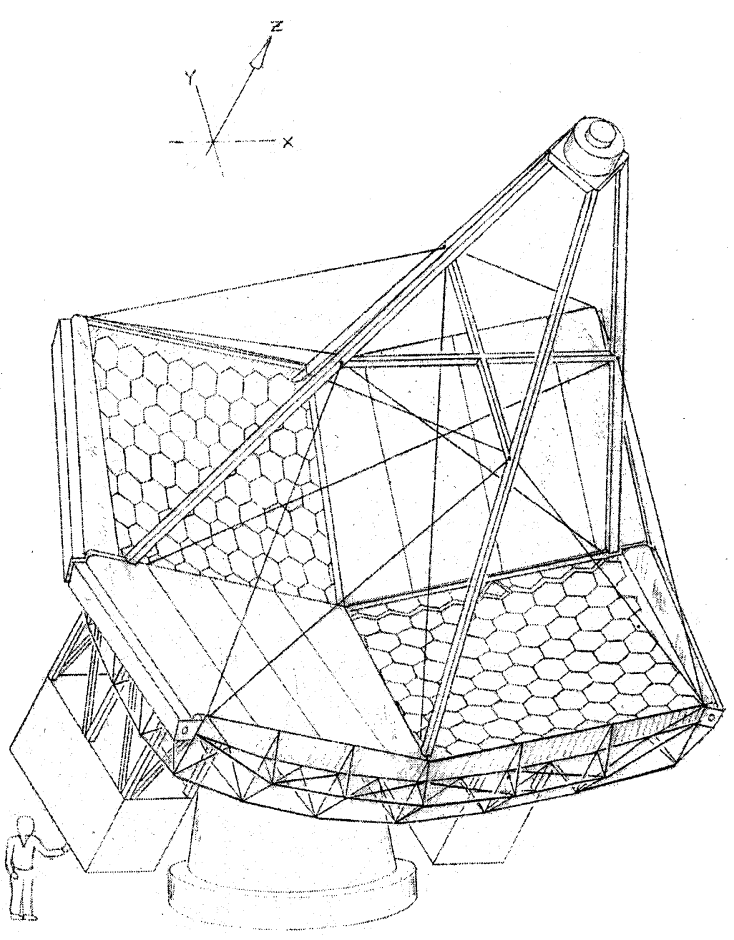

Fig. 9. Engineering sketch of VERITAS telescope based on conceptual design with covers retracted on upper left and lower right quadrants. The telescopes will not initially be populated by mirrors in corners. Sketch by Hoffman.

and/or lookup tables), are $0.02 / 0.04^{\circ}, 0.01 / 0.05^{\circ}$, and $0.02 / 0.05^{\circ}$, respectively for wind speeds up to 20 m.p.h. Azimuth slew speed will be $1 \%$ s and elevation slew speed will be $0.5^{\circ} / \mathrm{s}$. The total weight of the OSS, mirrors, counterweights and the detector is estimated to be $16,000 \mathrm{~kg}$. Commercially available positioners from radio and radar antenna manufacturers can support this weight and meet the pointing specifications.

When not in use, the mirrors on the reflector will be covered by four "window-blind" covers which will be mounted on the sides of the OSS.

\subsection{Electronic camera}

\subsubsection{Outline}

The electronic camera for each VERITAS telescope is similar to previous imaging atmospheric Cherenkov detectors [7] but with some significant improvements. An outline of the major components of the camera is shown in Fig. 10.

The array trigger is formed from a combination of individual telescope triggers at the central trigger. The trigger is designed to record all $\gamma$-ray events that contain enough light to be usefully identified as $\gamma$-ray events (reconstruction threshold) while limiting the trigger rate to a manageable level.

When a readout trigger decision has been made for an individual telescope, a sub-array of telescopes or the full array, the control electronics loads the appropriate section of the flash analogto-digital converter (FADC) memory of those signal channels with hits onto a data bus which is read out by the controller and stored in reflective memory modules. This information is read out by local CPUs and merged into event data for the telescope. The individual telescope data is transmitted to the central station, merged with data from the other telescopes and stored. A centrally generated optical pulse is used to synchronize individual electronic channels and the time tagging of events across the array.

Some of the individual elements of the VERITAS electronic cameras are discussed briefly below.

\subsubsection{Front-end electronics}

4.3.2.1. Photomultiplier tubes. A low noise, high gain $\left(>10^{6}\right)$, photon counting detector, with fast time response (risetimes $<2.5 \mathrm{~ns}$ ) matched to the intrinsic temporal width of the $\gamma$-ray induced air shower is necessary to increase the signal-to-noise ratio and to operate VERITAS at the lowest possible energy. These requirements are presently satisfied only by PMTs. The spacing between the centers of the PMTs corresponds to a focal plane angular distance of $0.15^{\circ}$. The active light collection diameter of the PMTs is $25 \mathrm{~mm}$. The collection efficiency is increased by the use of light concentrators. Tests have identified the Hamamatsu R7065, Electron Tube 9142W and Phillips Photonis XP2900 as PMTs which satisfy the VERITAS requirements.

4.3.2.2. High voltage supply. Modular commercial high voltage supplies will be used so that each PMT has a separately programmable high voltage. 


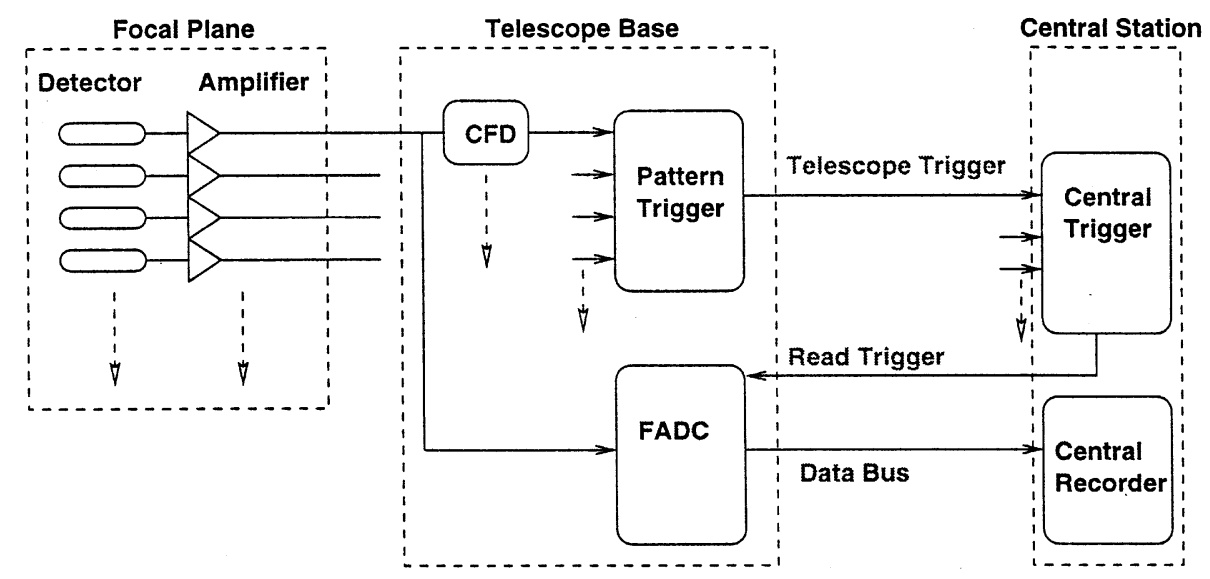

Fig. 10. Outline of electronics. The PMT signals are amplified in the camera head and transmitted to the electronics located in a building near the telescope base. The input analog signals are split with one output fed to fast CFDs to form the initial trigger by detecting coincidences on a time scale from 5 to $15 \mathrm{~ns}$. The outputs from these discriminators are fed to a local pattern trigger unit which tests for adjacent detector hits in the telescope. The other branch of the analog signal from each detector is fed to a FADC which digitizes the detector waveform into a circulating memory. This results in a digitized version of the signal pulse from each pixel. The telescope trigger signal is sent to the central trigger location where programmable delays are applied to the trigger signal from each telescope to correct for the difference in the time of arrival of the wavefront at the individual telescopes due to the source location in the sky.

These are used to adjust the PMT gains and to reduce the current draw when a bright star image falls directly on a specific PMT. A suitable unit which provides a $900 \mathrm{~V}$ swing in 48 channel modules is available from CAEN. The high voltage is supplied by cables attached to each PMT from a remote system crate. Commands to each telescope system are sent from the central station via Ethernet.

\subsubsection{Signal amplifiers. A custom circuit with a} linear amplifier is attached to each PMT in the focus box. This circuit is based on a standard integrated circuit amplifier chip, e.g., National Electronics Semiconductors CLC449 which have a bandwidth of $\sim 1 \mathrm{GHz}$. The targeted gain of the PMT and amplifier provides an output threshold signal level of $\sim 2 \mathrm{mV} /$ p.e. and a dynamic range of 1800. The amplifier also provides a secondary input to the data acquisition system which permits charge injection for calibration and diagnostic purposes during daytime.

4.3.2.4. Cabling. Pulses are transmitted from the focal plane PMTs to the telescope electronics through $40 \mathrm{~m}$ of high quality coaxial cable. This introduces some time dispersion into the signals. An analog optical fiber system with sufficient dynamic range (1:1000) is being tested on the Whipple $10 \mathrm{~m}$ telescope [30]. Fibers introduce no dispersion and are light-weight. If these tests are satisfactory optical fibers would be an attractive upgrade alternative to coaxial cabling.

\subsubsection{Trigger electronics}

VERITAS must trigger at low PMT threshold levels to achieve its low energy threshold, and with simple trigger schemes this would naturally lead to very high trigger rates. To limit the overall trigger rate and to keep the data acquisition deadtime to an acceptable level, a multi-level trigger scheme has been developed. This system keeps the overall trigger rate below $1 \mathrm{kHz}$. The four trigger levels are defined as

Level 1 (constant fraction discriminators): The outputs from the PMTs come directly to constant fraction discriminators (CFDs). The analog bandwidth of this system is $>500 \mathrm{MHz}$. The discriminator threshold and output pulse width are programmable by command. This module also has 
a short adjustable delay element to allow the timing in each channel to be matched to better than 2 ns. An analog fanout of the PMT signal is provided from this board to the FADC system. The CFDs constitute the level 1 trigger. The NSB produces PMT singles rates given by the dotted curve in Fig. 11. At low thresholds the dominant contribution to the trigger rate comes from fluctuations in the number of single p.e. produced by NSB. At higher thresholds the background rate is dominated by PMT afterpulsing. At a threshold of 4.2 p.e., the level 1 trigger rate is $\sim 1 \mathrm{MHz}$ for each channel.

Level 2 (pattern selection trigger): A hardware pattern trigger at each telescope based on level 1 triggers reduces the background by discriminating between photon-initiated shower images, which are compact, and background triggers caused by sky noise or afterpulsing, which have random locations in the camera plane. The dashed curve in Fig. 11 shows the expected rate of these back- ground events when the coincidence requirement is that at least three nearest neighbors in the 499 pixel camera are hit. The pattern selection trigger (PST) follows the scheme presently being used at the Whipple telescope [31].

Level 3 (array): The central station receives the level 2 triggers from each telescope. The level 3 trigger system selectively delays each level 2 signal to account for the wavefront orientation and determines if the overall array trigger condition is satisfied. The trigger condition depends on the array configuration and the observing strategy, but typically requires the time coincidence of several telescopes. The solid curve in Fig. 11 shows the expected background rates for this trigger if three of seven telescopes fire within $40 \mathrm{~ns}$ coincidence time. If the level 3 trigger condition is met, readout of the telescope event information is initiated.

Level 4: The background trigger rate can be reduced by demanding that the individual telescope trigger clusters conform to the predicted

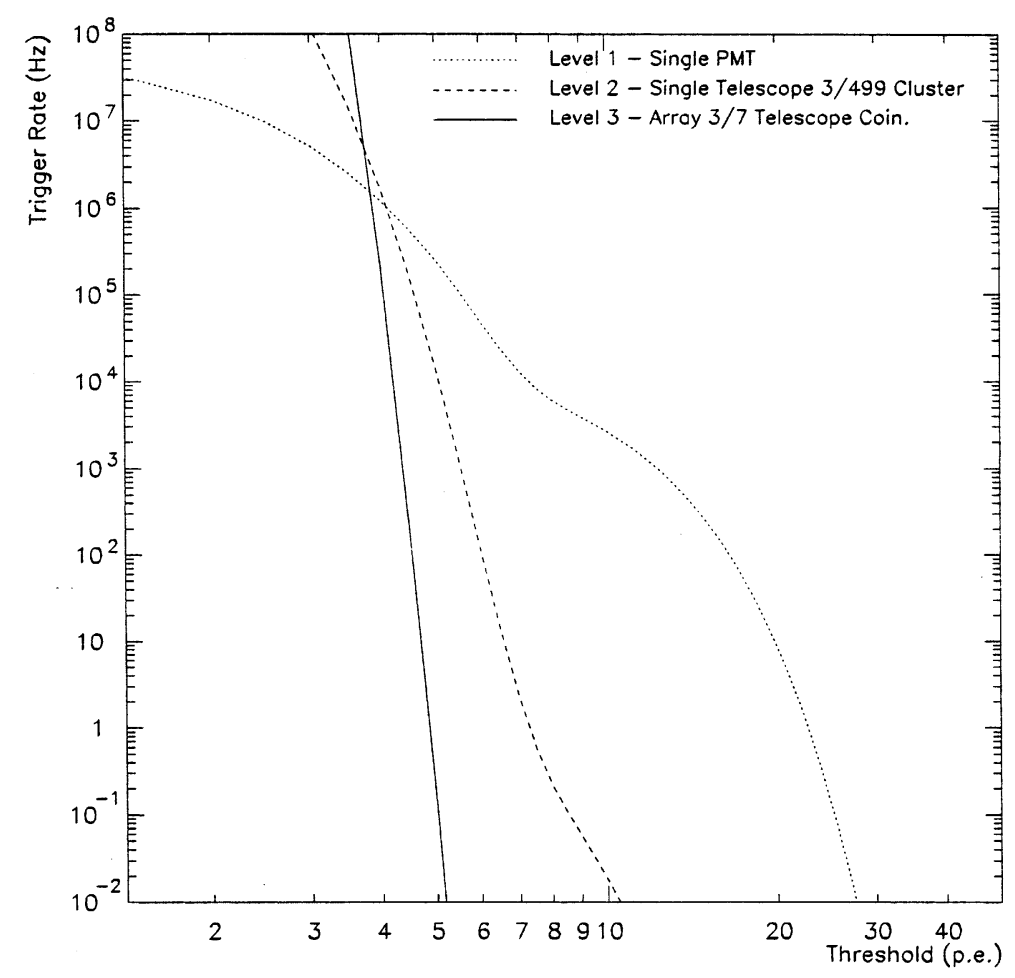

Fig. 11. Expected rates for the various trigger levels of VERITAS as a function of PMT threshold. The various curves are discussed in the text. 
parallactic displacement of the $\gamma$-ray images. Thus, a high level trigger, implemented either in hardware or in software, can be used to significantly suppress the hadronic background. This system is currently in the development stage.

\subsubsection{Signal recording}

4.3.4.1. Flash ADCs. The FADC provides a digitized version of the Cherenkov pulse waveform, giving the maximum information possible about the shape and time structure. These devices allow operation of the individual telescopes well into the NSB, eliminate the need for delay cables (minimizing signal dispersion), and allow real-time calibration of the PMT and signal cable propagation times. Also, the time structure of the pulse is expected to become wider near the edge of the Cherenkov light pool, and with the FADCs these effects can be corrected for in later analysis of the digitized pulses. The increasing availability of high bandwidth FADC chips for commercial purposes makes it possible to include a FADC in every detector channel of VERITAS. Commercial modules are expensive and VERITAS will use custom-built units. A prototype FADC system has been developed and tested on the Whipple telescope and appears to have all the necessary parameters for VERITAS. Each FADC channel uses a commercially available 8-bit FADC integrated circuit. This system has a sample rate of $500 \mathrm{MHz}$, a memory depth of $64 \mu \mathrm{s}$, and a novel autoranging gain switch that provides a dynamic range of 0 to 1020 with no loss of signal bandwidth [32].

4.3.4.2. Data acquisition. The data acquisition electronics are based largely around the VME standard electronic architecture: a fast VME backplane and distributed computation performed by local CPUs running a real-time operating system. Each controller will be connected, using fast, fiber-optic connections to a local workstation which in turn is connected to a central high speed switch connected to the central CPU. The central CPU will perform control and quicklook functions, further data compression and integration of the distributed data. This system will operate using cyclic and multi-thread processes, adhering to POSIX standards where possible in order to maintain future systems compatibility. Secondary systems to control mount movement and high voltage generation will be Pentium PCs running a UNIX-like operating system such as Linux. Communication will be via Ethernet connection. For a telescope readout rate of $\sim 1 \mathrm{kHz}$, each telescope is expected to have an average data flow rate of $\sim 1600 \mathrm{kbyte} / \mathrm{s}$, resulting in a $400 \mathrm{kbyte} / \mathrm{s}$ rate on any VME backplane. This rate includes real Cherenkov events and spurious background hits. The VERITAS data acquisition system will operate well within this limiting range.

\subsection{Calibration}

The seven telescope VERITAS array has substantially better energy resolution $(\Delta E / E \approx 15 \%)$ than the single Whipple $10 \mathrm{~m}$ telescope $(\approx 35 \%)$, thereby requiring a substantially improved calibration system. The maintenance of the larger number of data acquisition channels requires an automated system for both optical and electronic injection into the PMTs and preamplifiers. The widely-spaced array also requires special attention to details such as nightly calibration of telescope gains and multi-telescope event timing synchronization.

The angular resolution cannot be measured directly in the absence of test beam, but it can be verified by observations of a point source, e.g. an AGN.

The VERITAS calibration system has been designed to provide automated, redundant measurements of all relevant calibration parameters as well as to test system functionality automatically during the daytime. It consists of three different subsystems: optical injection, electronic or charge injection, and atmospheric monitoring. The optical injection system consists of a central pulse light source with variable optical attenuation which is distributed to all telescopes through uv-transmitting fiber optic cables. The optical pulses are used to illuminate the camera directly and to measure the mirror reflectivity by bouncing the light off the mirror surface before recording the optical pulse with the camera. The charge injection system injects a computer-adjustable pulse height and pulse width into each preamplifier input in order to 
measure electronic gain, as well as to verify each channel's operation. The charge injection system is located inside the camera, and is capable of triggering any possible combination of tubes by setting an internal 'mask'. This capability allows one to generate automatically various tube hit configurations to diagnose triggering capability of all four trigger levels. The atmospheric monitoring system consists of several radio-controlled light sources on the side of Mt. Hopkins as well as a Polaris monitor/star tracker telescope. Both the light sources and the star tracker/Polaris monitor use CCD cameras with UBVRI filter wheels to spectroscopically analyze the recorded light sources. The overall absolute calibration uncertainty for the combined calibration system will be $\approx 15 \%$, appropriate for the expected energy resolution of the VERITAS array.

\section{Scientific objectives}

\subsection{Active galactic nuclei}

The AGN detected at $\gamma$-ray energies are blazars which are generally radio-loud, flat-spectrum sources and radio and X-ray selected BL Lacertae
(BL Lac) objects. These sources are characterized by highly variable and predominantly non-thermal emission. Currently, no model for the production of $\gamma$-rays in the AGN is generally accepted. The two most popular models are those in which high energy electrons (known to be present in the jets from radio, optical, and X-ray observations) produce the $\gamma$-rays by inverse Compton scattering of low energy photons and those in which VHE protons produce the $\gamma$-rays by initiating cascades within the jets.

VHE observations of the blazars Markarian 421 (Mrk 421) and Markarian 501 (Mrk 501) have revealed extremely variable VHE emission (Fig. 12) [33,34]. Whipple and EGRET observations have also led to reformulations of blazar unification models based on intrinsic source luminosity rather than solely on the effects of orientation differences [35,36].

Despite these gains, there is much to learn about these enigmatic objects. VERITAS can help answer many of the unknowns concerning blazars, particularly when combined with lower energy observations by GLAST, X-ray and optical telescopes. The study of AGN will be approached from two perspectives: detailed studies involving observations of individual objects to reveal the

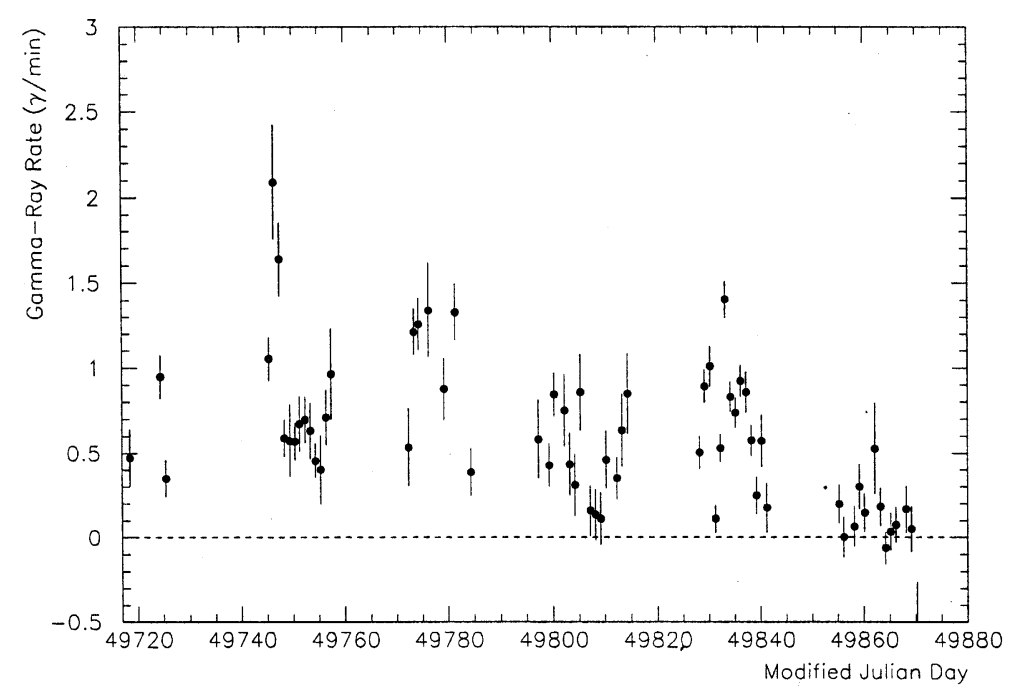

Fig. 12. Variability in $\gamma$-ray signal from Markarian 501 in 1997. 
physics operating in the AGN, and broad studies where more VHE sources are detected to illuminate the properties of different object classes.

\subsubsection{Detailed studies}

The large effective area of VERITAS enables accurate measurements of extremely rapid variations in the $\gamma$-ray flux as illustrated in Fig. 13. The left part of the figure shows Whipple observations of the fastest flare ever recorded at $\gamma$-ray energies. Although the flare was clearly detected, the structure of the flare may not have been fully resolved. The dashed curve in the figure is a hypothetical flux variation which matches the Whipple data. The right part of the figure shows a simulation of what VERITAS would detect above $200 \mathrm{GeV}$. All features of the flare are clearly resolved. VERITAS can be divided into two dedicated sub-arrays of 3 4 telescopes, with one sub-array observing a single object for as long as it is above the $30^{\circ}$ observation "horizon" for as many nights as a multiwavelength campaign lasts. Thus, VERITAS can provide more complete measurements of flaring activity and greatly increase the chance of seeing a wide range of activity in the objects studied. The sensitivity of VERITAS to short timescale variations will be an excellent match to X-ray and optical telescopes, permitting much improved measurements of energy-dependent variability.

\subsubsection{Broad studies}

The flux sensitivity of VERITAS will permit the detection of weaker sources of VHE emission. Its sensitivity at low energies will permit the viewing of objects further from Earth (the optical depth for pair production decreases rapidly with decreasing energy) and those objects which have spectral cut-offs below the sensitive range of existing Cherenkov telescopes. Based on the multiwavelength spectra of Mrk 421 and Mrk 501 and the spectral energy distributions of known BL Lacs, VERITAS will detect 30 or more X-ray selected BL Lacs.

EGRET sources associated with AGN now number at least 67 [37] and span a range of redshifts from $z=0.03$ to 2.28 . However only six AGN have been detected above $300 \mathrm{GeV}$. The most likely causes of this disparity are intrinsic cut-offs in the source spectra and, for the more distant sources, attenuation of the $\mathrm{TeV} \gamma$-rays by
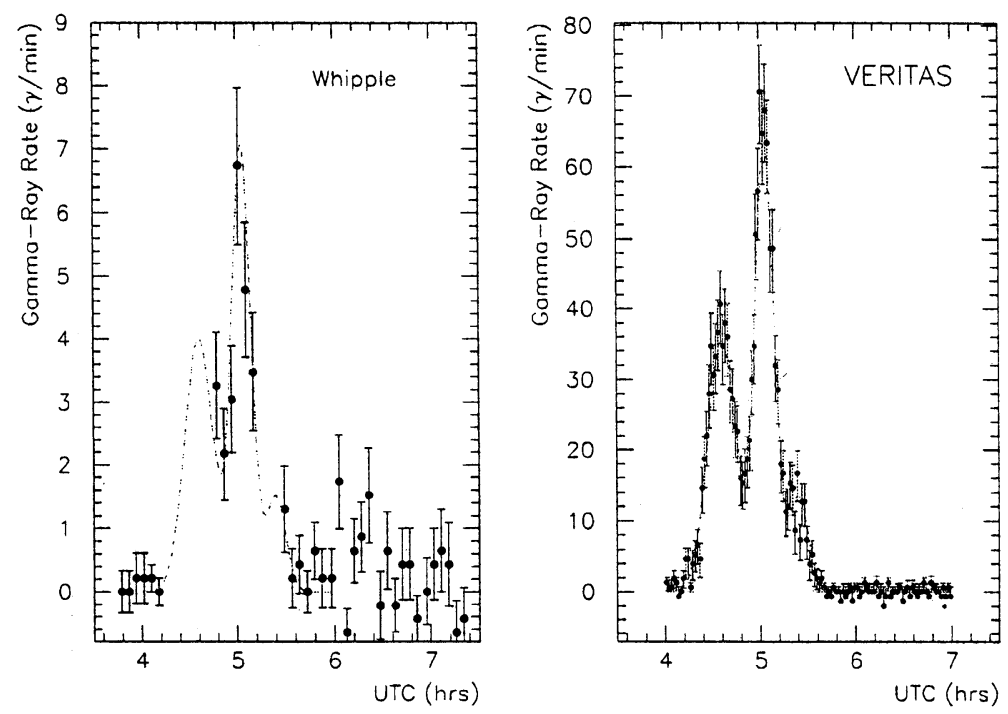

Fig. 13. Left: Whipple observations of a rapid flare from Mrk 421 on 1996, May 15. The superimposed dashed curve is a possible intrinsic flux variation which is consistent with the VHE data. Right: Simulated response of VERITAS to such a flare above $200 \mathrm{GeV}$. 
pair production with background IR radiation. The low energy threshold and good sensitivity of VERITAS will overcome these hurdles and lead to the detection of approximately 15 of the EGRET AGN, more if GLAST or all-sky X-ray monitors indicate when these objects are in high emission states. VERITAS is in a unique position to make these measurements, providing better photon statistics than space-based telescopes at these energies and superior energy resolution and sensitivity relative to other ground-based telescopes.

\subsection{Shell-type supernova remnants}

SNRs are widely believed to be the sources of hadronic cosmic rays up to energies of approximately $Z \times 10^{14} \mathrm{eV}$, where $Z$ is the nuclear charge of the particle. However, a clear indication for the acceleration of hadronic particles in SNRs is still missing. The existence of energetic electrons is well known from observations of synchrotron emission at radio and X-ray energies (e.g., Ref. [38]). Recently, the detection of $\mathrm{TeV} \gamma$-rays from the shelltype remnants, SN 1006, RXJ 1713.7-3946 and Cassiopeia A has been reported [5]. When combined with X-ray and radio observations, the VHE $\gamma$-ray observations provide a means of resolving the various contributions (proton-proton collisions or electron bremsstrahlung or inverse Compton scattering). The combined observations can also give information about SNR shell environments such as the maximum particle energy and magnetic field. Both quantities are important, but unknown, parameters in shock acceleration theories.

Fig. 14 shows the EGRET measurements and Whipple upper limits for the SNR IC 443. These results already eliminate much of the allowed parameter space for $\gamma$-ray emission from these objects (from hadrons and electrons), and begin to raise some questions about the validity of current models for the objects studied and even for the SNR origin of cosmic rays.

For a typical SNR luminosity and angular extent, VERITAS should be able to detect objects within $4 \mathrm{kpc}$ of Earth according to one popular model of $\gamma$-ray production by hadronic interac- tions [39]. Approximately, twenty shell-type SNRs with known distances lie within this distance range, permitting VERITAS to investigate which characteristics of SNR are necessary for them to be sites of particle acceleration.

\subsection{Diffuse galactic $\gamma$-ray emission}

High energy $\gamma$-rays traverse the Galaxy without significant attenuation implying that the diffuse emission probes high energy processes in the Galaxy as a whole. EGRET studies [40] show generally good agreement with detailed models in both spatial and spectral features. A striking exception is that there is a $40 \%$ excess in measured flux at the highest EGRET energies, and the measured spectrum systematically rises above predictions. In some models, e.g., Refs. [41,42] this is attributed to inverse Compton scattering from SNR electrons injected with a hard spectral index, although this appears to be in contradiction to upper limits at $\mathrm{TeV}$ energies [43]. Detailed spectral studies in the $\mathrm{GeV} / \mathrm{TeV}$ energy bands are necessary to understand the discrepancies.

\subsection{Compact galactic objects}

\subsubsection{Pulsar-powered nebulae}

With the flux sensitivity of VERITAS it will be possible to detect Crab Nebula-like objects anywhere within the Galaxy if their declination is $>-28^{\circ}$. The energy resolution and broader energy coverage will significantly improve spectral measurements leading to accurate estimates of the nebular magnetic field and maximum electron energy, and to more detailed tests of $\gamma$-ray emission models. With its excellent angular resolution, VERITAS may even resolve the GeV-TeV emission region of nearby objects like the Crab Nebula.

\subsection{2. $\gamma$-ray pulsars}

Attenuation of $\gamma$-rays by pair-production interactions in the intense magnetic field near pulsars leads to a super-exponential cut-off in the spectra predicted by polar cap models. Because the outer 


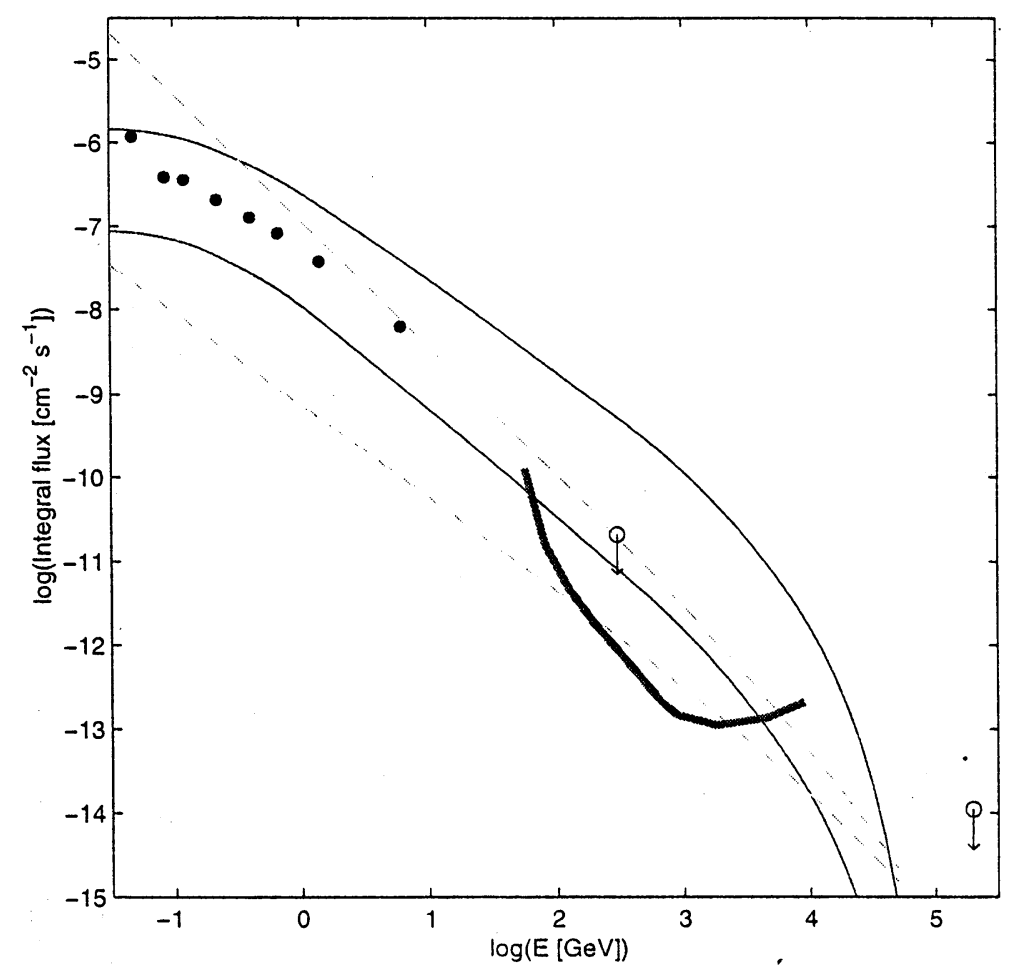

Fig. 14. Predicted $\gamma$-ray spectra in the shell-type SNR IC 443. The region between the solid lines depicts the spectra from hadronic interactions for a range of model parameters. The region between the dashed lines is the predicted inverse-Compton spectra for $B$ between 20 and $50 \mu \mathrm{G}$ and the range of flux normalizations and electron spectra allowed by the X-ray observations. EGRET data points (filled circles) and the current upper limits (open circles) from Whipple and the Cygnus air shower array are shown. The sensitivity of VERITAS for a $50 \mathrm{~h}$ observation of this object is indicated by the thick curve.

gap models do not predict such sharp cut-offs, the detection of pulsed $\mathrm{GeV}-\mathrm{TeV} \gamma$-rays may be decisive in favoring the outer gap model over the polar cap model. The excellent sensitivity and energy resolution of VERITAS will allow spectral measurements to be conducted even though these objects are expected to have rapidly falling spectra in the $\mathrm{GeV}-\mathrm{TeV}$ range.

\subsubsection{Unidentified galactic EGRET sources}

A legacy of EGRET is 170 unidentified sources, many of which are in the Galactic plane [37]. The positional uncertainty of these sources make identifications with sources at longer wavelengths unlikely. VERITAS will have the sensitivity and low energy threshold necessary to detect many of these objects if their spectra do not cut-off above $10 \mathrm{GeV}$. Detailed studies with the excellent source location capability of VERITAS could lead to identifications with objects at longer wavelengths.

\subsubsection{Galactic plane survey}

Because of its flux sensitivity and large FOV, one of the first priorities of VERITAS will be to conduct a sensitive survey in the $100 \mathrm{GeV}-10 \mathrm{TeV}$ energy range. To maximize the scientific returns, the first task will be to survey the region near the Galactic plane. VERITAS can observe the plane over the longitude range $0^{\circ}<l<245^{\circ}$, but the initial survey will concentrate on the denser regions. For an 80-night survey of the region 
$0^{\circ}<l<85^{\circ}$, VERITAS will be sensitive to fluxes down to $\sim 0.02 \mathrm{Crab}$, above $300 \mathrm{GeV}$.

\subsection{Cosmic ray composition}

VERITAS, with its fine pixellation, large mirror area, and multi-telescope image measurement can measure the Cherenkov light emitted by the primary cosmic ray nucleus before it interacts with the atmosphere, thereby providing a high-resolution $(\Delta Z / Z<5 \%$ for $Z>10)$ charge measurement of cosmic rays around the knee of the all-particle spectrum [44]. This measurement will be essentially independent of any assumed nuclear interaction model; therefore VERITAS can provide a tagged nuclear beam that will determine the true airnucleus interaction characteristics as a function of primary charge and energy. This should provide a method to eliminate interaction model-dependent biases in the interpretation of these results, as well as allow other experiments to use the experimentally determined interaction model to reanalyze their data. In addition, VERITAS will be sensitive to nuclei heavier than iron in the PeV energy region, to exotic particle states such as relativistic magnetic monopoles [45] or strange quark matter [46].

\subsection{Background infrared radiation}

In traversing intergalactic distances, $\gamma$-rays can be absorbed by photon-photon pair production on background photon fields [47]. For VHE photons, the most important interaction is with IR fields. Measurements of VHE spectra from extragalactic sources permit indirect investigation into the nature of the IR background field because distortions in the $\gamma$-ray spectrum due to pair production depend on the density spectrum of the background field. VHE spectra from Mrk 421 and Mrk 501 have already been used to set upper limits on the IR background from $0.025 \mathrm{eV}$ to $0.3 \mathrm{eV}$ [48-51]. At some wavelengths, these limits are as much as an order of magnitude below the upper limits set by the DIRBE/COBE satellite (see Fig. 15 ). The current limits on the IR density are $\sim 5$ to
10 times higher than predicted from galaxy formation and evolution (e.g., Refs. [52,53]). However, with better spectral measurements of existing sources and detections of more objects, particularly at higher redshift, VERITAS can substantially improve these limits, and may eventually detect the effects of the IR field.

\section{7. $\gamma$-ray bursts}

The sources and mechanism for producing GRBs remain unknown. The detection by EGRET of an $18 \mathrm{GeV}$ photon from a GRB more than 90 min after the burst was detected by BATSE [54] demonstrates that high energy $\gamma$-rays play an important role in the energetics of GRBs. Also, because the high energy emission can be delayed, it can be pursued with rapid follow-up observations. More recently, the Milagro Collaboration has reported a weak $(\sim 3 \sigma)$ detection of $\mathrm{TeV}$ emission from a GRB [55] raising the possibility that $\mathrm{TeV}$ photons may carry a significant fraction of the energy emitted in a GRB. Because of its low energy threshold, VERITAS will be able to see bursts with hard spectra out to $z \sim 1$ or more.

\subsection{Neutralino annihilation in the galactic center}

Current astrophysical data would appear to indicate the need for a cold dark matter component with $\Omega \approx 0.3$ (see Refs. [56-58]). A good candidate for this component is the neutralino, the lightest stable supersymmetric particle. If neutralinos do comprise the dark matter and are concentrated near very massive astrophysical objects like the center of the Galaxy, their direct annihilation to $\gamma$-rays should produce a unique signal not easily mimicked by other astrophysical processes: a monoenergetic annihilation line with mean energy equal to the neutralino mass. Cosmological constraints and limits from accelerator experiments restrict the neutralino mass to the range 30 GeV-3 TeV. Thus, VERITAS and GLAST together will allow a sensitive search over the entire allowed neutralino mass range. Recent estimates of the annihilation line flux for neutralinos at the galactic center [59] using a galactic model with 


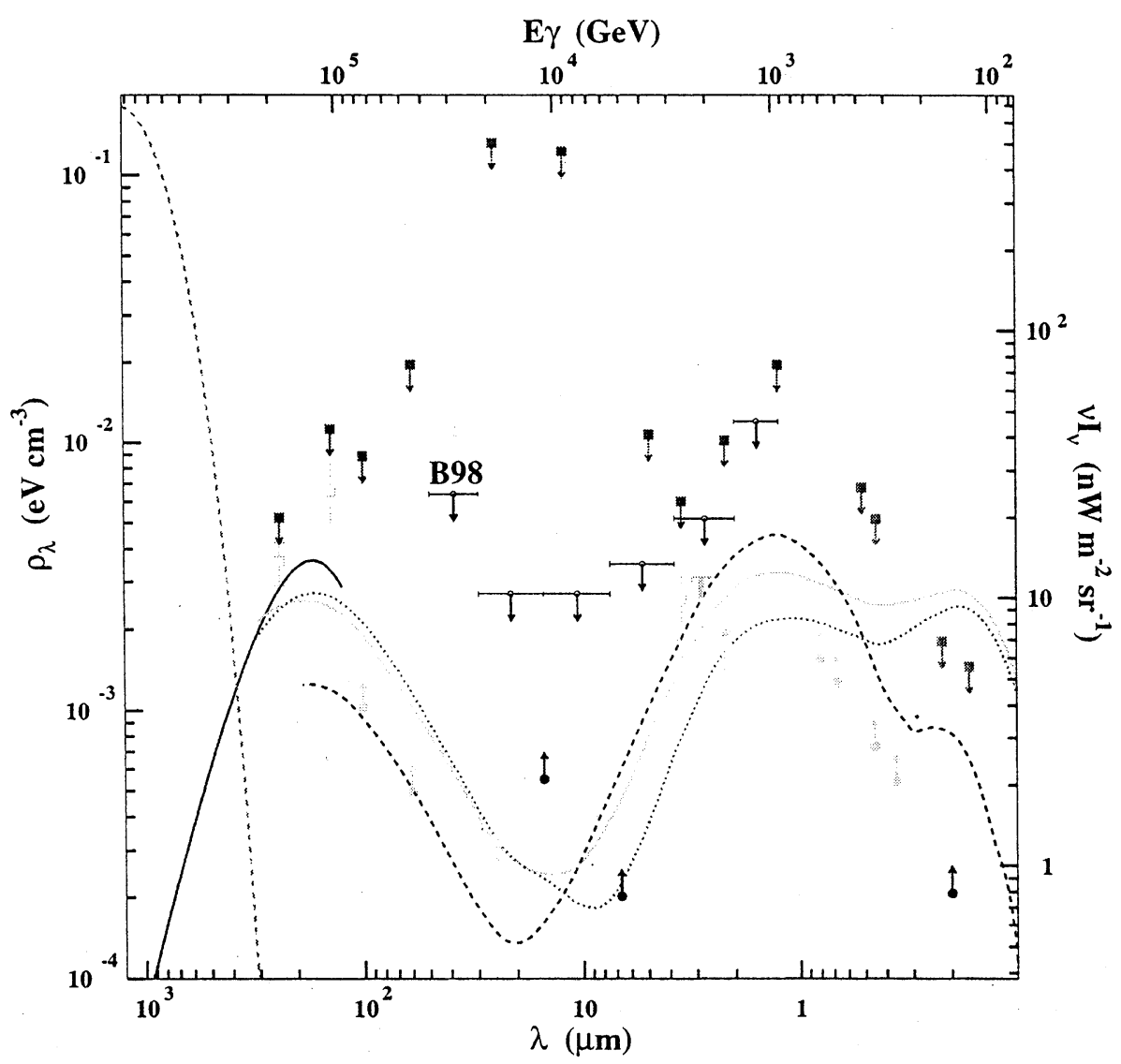

Fig. 15. Limits on the extragalactic IR background derived from DIRBE and other experiments. The limits from TeV measurements are shown as broad arrows while the predictions of theoretical models are shown as dotted lines.

central cusps in the density distribution of the dark matter halos [60] predict a $\gamma$-ray signal which may be of sufficient intensity to be detected with VERITAS and GLAST. The better sensitivity and lower energy threshold of VERITAS will be critical to covering a broad part of the allowed range of neutralino and dark matter parameters.

\subsection{Quantum gravity}

Quantum gravity can manifest itself as an effective energy dependence to the velocity of light in a vacuum. This dependence is caused by propagation through a gravitational medium containing quantum fluctuations on distance scales near the Planck length ( $\left.\simeq 10^{-33} \mathrm{~cm}\right)$ (e.g., Ref. [61]).
If the quantum gravity correction to vacuum refractive index exists, it should appear at the energy scale comparable to Planck mass $\left(\approx 10^{19}\right.$ $\mathrm{GeV})$. Recent work within the context of string theory indicates, however, that the quantum gravity scale may occur at a much lower energy, perhaps as low as $10^{16} \mathrm{GeV}$ [62]. TeV observations of variable emission from astrophysical objects provide a means of searching for the effects of quantum gravity [63]. VERITAS will significantly improve short timescale variability measurements and also will detect more distant objects. Variability on short timescales from sources at $z>0.1$ would be sensitive to quantum gravity effects and would provide a test of the validity of Lorentz symmetry at energies within a factor of five of the Planck mass (in some models). 


\subsection{Primordial black holes}

Primordial black holes, if they exist, should emit a burst of radiation in the final stages of their evaporation (e.g., Ref. [64]). In the standard model of particle physics, this last burst of radiation should release about $10^{30} \mathrm{erg}$ in $1 \mathrm{~s}$ with the energy distribution peaked near $1 \mathrm{TeV}$ [65]. In two years of operation VERITAS would be able to reach a sensitivity level for this type of evaporation of $700 \mathrm{pc}^{-3} \mathrm{yr}^{-1}$. More extreme models (e.g., Ref. [66]) would produce lower energy events on much shorter time-scales which may be detectable with VERITAS using a special trigger and the flash ADC system [67].

\section{Status of VHE $\gamma$-ray astronomy}

There are seven groups now using the atmospheric Cherenkov imaging technique [68]. These techniques are relatively mature and the results from simultaneous observations, with different telescopes of the same source are consistent [69]. Vigorous observing programs are now in place at all of these facilities. An important milestone for the field has been reached in that both galactic and extragalactic sources have been reliably detected [5].

To exploit fully the potential of ground-based $\gamma$-ray astronomy the detection techniques must be improved. This will happen by extending the energy coverage of the technique and by increasing its flux sensitivity. Ideally one would like to do both, but in practice there must be trade-offs. Reduced energy thresholds can be achieved by the use of larger, but cruder, mirrors. This approach is currently being exploited using existing arrays of solar heliostats: STACEE [70], CELESTE [71], Solar-2 [72], GRAAL [73]. These projects may achieve thresholds as low as $20-30 \mathrm{GeV}$ where they will effectively fill the current gap in the $\gamma$-ray spectrum from 20 to $200 \mathrm{GeV}$ but with poor energy resolution and small fields of view. A European project (MAGIC) [74] to build a $17 \mathrm{~m}$ aperture telescope is now under way and a similar project, MACE has been proposed in India [75]. Ultimately this gap will be covered by GLAST with weaker point source sensitivity at the higher energies. This next generation $\gamma$-ray space telescope is scheduled for launch in 2005 by an international collaboration [77]. Extension to higher energies $(>10 \mathrm{TeV})$ can be achieved by atmospheric Cherenkov telescopes working at large zenith angles and by particle arrays at very high altitudes. The MILAGRO water Cherenkov detector in New Mexico [78] operates $24 \mathrm{~h}$ a day with a large FOV and has good sensitivity to GRBs and transients.

The primary objective of VERITAS will be to have high sensitivity in the $100 \mathrm{GeV}$ to $10 \mathrm{TeV}$ range. The German-French HESS (initially four and eventually perhaps sixteen $12 \mathrm{~m}$ class telescopes) will be built in Namibia $[19,76]$ and the Japanese CANGAROO-III array (with four telescopes in Australia) [79,80] will have similar objectives for observations in the southern hemisphere. MAGIC, HESS and CANGAROO are approved projects with target completion dates in 2002-2004. The arrays will exploit the high sensitivity of the atmospheric Cherenkov imaging technique and the high selectivity of the array approach. The relative flux sensitivities as a function of energy are shown in Fig. 16, where the sensitivities of the wide field detectors are for one year and the ACT are for $50 \mathrm{~h}$; in all cases a $5 \sigma$ point source detection is required. The flux sensitivity of HEGRA, CAT and CANGAROO-II are very similar to that of Whipple; similarly the flux sensitivity of HESS and CANGAROO-III are anticipated to be similar to that of the simulated sensitivity of VERITAS. Hence the integral flux sensitivities shown in Fig. 16 for Whipple and VERITAS are representative of the other observatories.

It is apparent from this figure that, on the low energy side, VERITAS will complement the GLAST mission and will overlap with STACEE and CELESTE. At its highest energy it will overlap with the Tibet air shower array [81].

It will cover the same energy range as MILAGRO but with greater flux sensitivity. As a northern hemisphere telescope VERITAS will complement the coverage of neutrino sources to be discovered by AMANDA and ICE CUBE at the South Pole. 


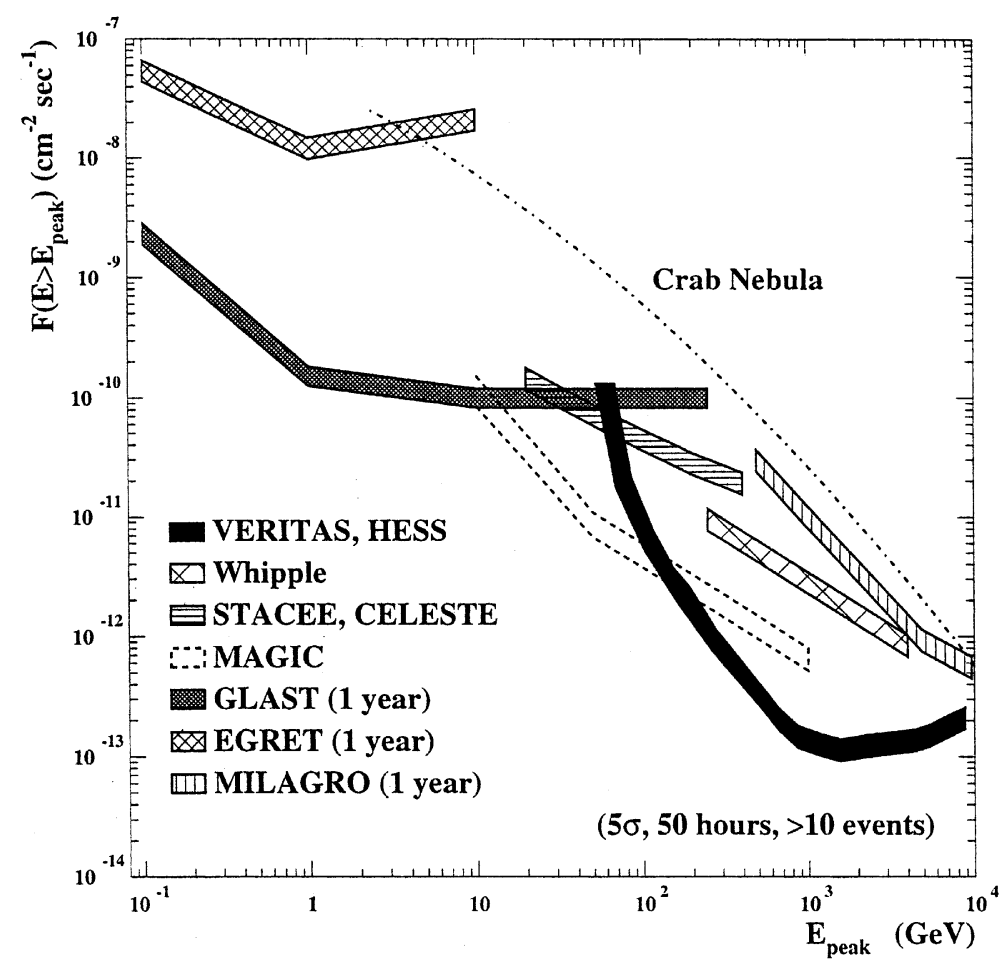

Fig. 16. Comparison of the point source sensitivity of VERITAS to Whipple, MAGIC, CELESTE/STACEE; GLAST, EGRET and MILAGRO. The sensitivity of MAGIC is based on the availability of new technologies, e.g., high quantum efficiency PMTs, not assumed in the other experiments. EGRET, GLAST and MILAGRO are wide field instruments and therefore ideally suited for all sky surveys. The turn-up in the VERITAS sensitivity at higher energies is primarily caused by the limited field of view of the cameras and the requirement of VERITAS detecting at least 10 photons.

\section{Acknowledgements}

This work was supported in part by grants from the US Department of Energy, the National Science Foundation, the Smithsonian Institution, PPARC in the U.K. and Enterprise-Ireland in Ireland. The referees are thanked for helpful suggestions.

\section{References}

[1] R.A. Ong, Phys. Rep. 305 (1998) 93.

[2] F.A. Aharonian, C.W. Akerlof, Ann. Rev. Nucl. Part. Sci. 47 (1997) 273.

[3] C.M. Hoffman et al., Rev. Mod. Phys. 71 (1999) 897.

[4] M. Catanese, T.C. Weekes, PASP 111 (1999) 1193.

[5] T.C. Weekes, GeV-TeV Astrophysics: Towards a Major Atmospheric Cherenkov Detector VI (Snowbird), 1999, p. 3 (astro-ph/9910394).
[6] T.C. Weekes, K.E. Turver, Proc. 12th ESLAB Symp. (Frascati), 1977, p. 279.

[7] M.F. Cawley et al., Exp. Astron. 1 (1990) 173.

[8] T.C. Weekes et al., ApJ 342 (1989) 370.

[9] G. Vacanti et al., ApJ 377 (1991) 467.

[10] A. Barrau et al., NIM A 416 (1998) 278.

[11] T. Hara et al., NIM A 332 (1993) 300.

[12] A. Konopelko et al., Astropart. Phys. 11 (1999) 135.

[13] S. Aiso et al., in: M.S. Potgeier et al. (Eds.), Proc. 25th ICRC (Durban), vol. 5, 1997, p. 373.

[14] P.M. Chadwick et al., GeV-TeV Astrophysics: Towards a Major Atmospheric Cherenkov Detector VI (Snowbird), 1999 , p. 86.

[15] B.M. Vladimirsky et al., Proc. Workshop on VHE Gamma Ray Astronomy, Crimea, April 1989, p. 21.

[16] S.I. Nikolsky et al., Proc. Workshop on VHE Gamma Ray Astronomy, Crimea, April 1989, p. 11.

[17] C.L. Bhat et al., Proc. Workshop on VHE Gamma Ray Astronomy, (Kruger Park), 1997, p. 196.

[18] F. Krennrich et al., Astropart. Phys. 8 (1998) 213.

[19] A. Konopelko, TeV astrophysics of extragalactic sources, Astropart. Phys. 11 (1999) 263. 
[20] D.A. Lewis, Exp. Astron. 1 (1990) 213.

[21] T.C. Weekes, Proc. of Workshop on Cosmic Ray and High Energy Gamma Ray Experiments for the Space Station Era, Baton Rouge, October 1984, 1985, p. 497.

[22] V.V. Vassiliev, Astropart. Phys. 11 (1999) 247, astro-ph/ 9908088.

[23] V.V. Vassiliev, Proc. 26th I.C.R.C., vol. 4, Salt Lake City, July 1999, p. 299.

[24] T.C. Weekes, VERITAS Proposal to U.S.D.O.E., unpublished.

[25] F. Krennrich et al., ApJ 511 (1999) 149.

[26] J.H. Buckley et al., AsA 329 (1998) 639.

[27] A.M. Hillas, Space Sci. Rev. 75 (1996) 117.

[28] G. Mohanty et al., Astropart. Phys. 9 (1998) 15.

[29] J.M. Davies, E.S. Cotton, J. Solar Energy Sci. Eng. 1 (1957) 16.

[30] I. de la Calle, Proc. 27th I.C.R.C., Hamburg, 2001, p. 2904.

[31] S. Bradbury et al., Proc. 26th I.C.R.C., vol. 4, Salt Lake City, 1999, p. 263.

[32] J.H. Buckley et al., Proc. 26th I.C.R.C., vol. 4, Salt Lake City, August 1999, p. 267.

[33] J. Quinn et al., ApJ 518 (1998) 693.

[34] J.A. Gaidos et al., Nature 383 (1996) 319.

[35] G. Ghisellini, TeV Astrophysics of Extragalactic Sources, Astropart. Phys. 11 (1999) 11.

[36] M. Georganopoulos, A.P. Marscher, Ap J 506 (1998) 621.

[37] R.C. Hartman et al., ApJ S 123 (1999) 79.

[38] M. Koyama et al., Nature 378 (1995) 255.

[39] L.O'C. Drury, F.A. Aharonian, H.J. Völk, AsA 287 (1994) 959.

[40] S.D. Hunter et al., ApJ 481 (1997) 205.

[41] M. Pohl, J.A. Esposito, ApJ 507 (1998) 327.

[42] I.V. Moskalenko, A.W. Strong, ApJ 528 (2000) 357.

[43] S. Lebohec et al., ApJ 539 (2000) 209.

[44] D. Kieda, S.P. Swordy, S.P. Wakely, Astropart. Phys. 15 (2001) 287.

[45] S.D. Wick et al., Astropart. Phys., submitted for publication.

[46] S. Banerjee et al., Phys. Rev. Lett. 85 (2000) 1384.

[47] R.P. Gould, G.P. Schréder, Phys. Rev. 155 (1967) 1408.

[48] F.W. Stecker, O.C. de Jager, AsA 334 (1998) L85.

[49] E. Dwek, J. Slavin, ApJ 436 (1992) 696.

[50] S.D. Biller et al., Phys. Rev. Lett. 80 (1998) 2992.

[51] V.V. Vassiliev, Astropart. Phys. 12 (2001) 217.

[52] J.R. Primack et al., TeV Astrophysics of Extragalactic Sources, Astropart. Phys. (1999) 11, 93.

[53] M.A. Malkan, F.W. Stecker, ApJ 496 (1998) 13.

[54] K. Hurley, Nature 372 (1994) 652.
[55] J.E. McEnery et al., GeV-TeV Astrophysics: Towards a Major Atmospheric Cherenkov Detector VI (Snowbird), 1999 , p. 243 (astro-ph/9910549).

[56] E. Gawiser, J. Silk, Science 280 (1998) 1405.

[57] J.R. Primack, Science 280 (1998) 1398.

[58] M. Kamionkowski, Science 280 (1998) 1397.

[59] L. Bergström, P. Ullio, J.H. Buckley, Astropart. Phys. 9 (1998) 137.

[60] J.F. Navarro, C.S. Frenk, S.D.M. White, ApJ 462 (1996) 563.

[61] G. Amelino-Camelia et al., Nature 383 (1998) 319.

[62] E. Witten, Nucl. Phys. B 471 (1996) 135.

[63] S.D. Biller et al., Phys. Rev. Lett. 83 (1999) 2108.

[64] D.N. Page, S.W. Hawking, ApJ 206 (1976) 1.

[65] F. Halzen et al., Nature 353 (1991) 807.

[66] R. Hagedorn, Nuovo Cim., LVI A Nr. 4 (1968) 1027.

[67] F. Krennrich, S. Le Bohec, T.C. Weekes, ApJ 529 (2001) 506.

[68] T.C. Weekes, Proc. of Symposium on VHE Gamma-ray Astronomy, Heidelberg, June 2000, AIP Conference Proceedings, Vol. 558, 2001, p. 15.

[69] R.J. Protheroe et al., in: M.S. Potgeier et al. (Eds.), Proc. 25th ICRC (Durban), vol. 8, 1997, p. 317.

[70] M.C. Chantell et al., NIM A 408 (1998) 468.

[71] B. Giebels et al., NIM A 412 (1998) 329.

[72] J. Zweerink et al., Proc. 26th I.C.R.C., vol. 4, Salt Lake City, August 1999, p. 223.

[73] F. Arqueros, 26th I.C.R.C., vol. 5, Salt Lake City, August 1999 , p. 215.

[74] E. Lorenz, GeV-TeV Astrophysics: Towards a Major Atmospheric Cherenkov Detector VI (Snowbird), 1999, p. 510 .

[75] C.L. Bhat et al., Proc. of Symposium on VHE Gamma-ray Astronomy, Heidelberg, June 2000, AIP Conference Proceedings, Vol. 558, 2001, p. 582.

[76] W. Hofmann, GeV-TeV Astrophysics: Towards a Major Atmospheric Cherenkov Detector VI (Snowbird), 1999, p. 500 .

[77] N. Gehrels, P. Michelson, TeV Astrophysics of Extragalactic Sources, Astropart. Phys. 11 (1999) 277.

[78] G. Sinnis et al., Nucl. Phys. B (Proc. Suppl.), 43 (1995) 141.

[79] Y. Matsubara in: O.C. de Jager (Ed.), Towards a Major Atmospheric Čerenkov Detector V (Kruger Park), 1997, p. 447.

[80] M. Mori et al., GeV-TeV Astrophysics: Towards a Major Atmospheric Cherenkov Detector VI (Snowbird), 1999, p. 485.

[81] M. Amenomori et al., in: M.S. Potgeier et al. (Eds.), Proc. 25th ICRC (Durban), vol. 5, 1997, p. 245. 